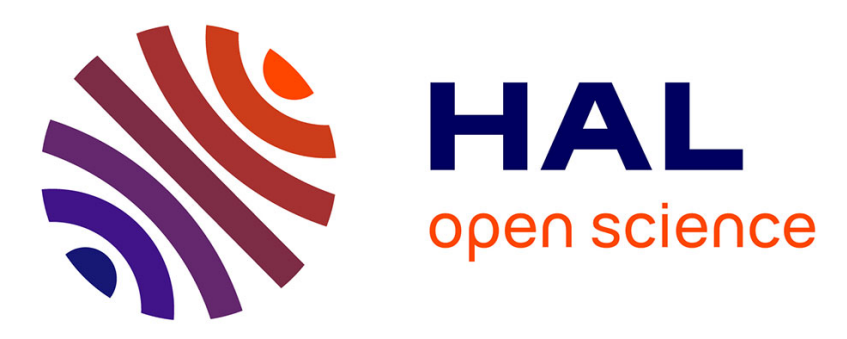

\title{
A non-conformal eXtended Finite Element approach: Integral matching Xfem
}

Elie Chahine, Patrick Laborde, Yves Renard

\section{To cite this version:}

Elie Chahine, Patrick Laborde, Yves Renard. A non-conformal eXtended Finite Element approach: Integral matching Xfem. Applied Numerical Mathematics, 2011, 61, pp.322-343. 10.1016/j.apnum.2010.10.009 . hal-00690453

\section{HAL Id: hal-00690453 https://hal.science/hal-00690453}

Submitted on 26 Apr 2018

HAL is a multi-disciplinary open access archive for the deposit and dissemination of scientific research documents, whether they are published or not. The documents may come from teaching and research institutions in France or abroad, or from public or private research centers.
L'archive ouverte pluridisciplinaire HAL, est destinée au dépôt et à la diffusion de documents scientifiques de niveau recherche, publiés ou non, émanant des établissements d'enseignement et de recherche français ou étrangers, des laboratoires publics ou privés. 


\title{
A non-conformal eXtended Finite Element approach: Integral matching Xfem
}

\author{
Elie Chahine ${ }^{\mathrm{a},}{ }^{*}$, Patrick Laborde ${ }^{\mathrm{b}}$, Yves Renard ${ }^{\mathrm{c}}$
}

\footnotetext{
a Paul Scherrer Institute, OHSA/B06, CH-5232 Villigen PSI, Switzerland

${ }^{\mathrm{b}}$ Université de Toulouse, CNRS, Université Paul Sabatier, Institut de Mathématiques de Toulouse, UMR 5219, F-31062 Toulouse Cedex 9, France

c Université de Lyon, CNRS, INSA-Lyon, ICJ UMR5208, LaMCoS UMR5259, F-69621, Villeurbanne, France
}

\begin{abstract}
This work is dedicated to the mathematical and numerical analysis of a new Xfem approach: the integral maching Xfem. It is known that the quality of the approximation and the convergence rate of Xfem type methods is broadly influenced by the transition layer between the singular enrichment area and the rest of the domain. In the presented method, this transition layer is replaced by an interface associated with an integral matching condition of mortar type. We prove an optimal convergence result for such a nonconformal approximation method and we perform some numerical experiments showing the advantages of the integral matching Xfem with respect to former Xfem approaches.
\end{abstract}

\section{Introduction}

The eXtended Finite Element Method (Xfem) is the subject of an increasing interest since its introduction in 1999 (see [21]). Some contributions intended to improve the performance of Xfem regarding its convergence rate and accuracy. In [1] and [17], a surface singular enrichment strategy was proposed that allowed to obtain an optimal convergence rate for Xfem. Meanwhile, this approach is expensive and ill-conditioned since the enrichment singular functions are assigned to every finite element node in a fixed area containing the crack tip. A special pre-conditioning strategy was used in [1] to treat this difficulty. On the other hand, it was proposed in [17] a "globalization" of the enrichment strategy that reduces the computational cost, but decreases the convergence rate. In order to improve the latter method, a generalized enrichment Xfem approach using a cut-off function was introduced in [6]. This method have an optimal convergence rate while reducing the computational cost of the standard Xfem with surface enrichment.

In $[6,22]$, the optimality of both Xfem with a cut-off function and Xfem with enrichment surface is established. However, it was pointed out in [17,6] that a certain loss in accuracy occurs for both methods. For Xfem with a cut-off function the loss in accuracy is due to the approximation of the cut-off function itself and for Xfem with enrichment surface the loss of accuracy occurs on the transition layer of the finite elements between the singular enrichment zone and the rest of the domain. It is also proved in [22] that Xfem with enrichment surface is always more accurate than Xfem with a cut-off function. The approximation over the partially enriched elements, the so-called blending elements, is less accurate than over

\footnotetext{
* Corresponding author.

E-mail addresses: elie.chahine@psi.ch, chahineelie@gmail.com (E. Chahine), laborde@math.ups-tlse.fr (P. Laborde), yves.renard@insa-lyon.fr (Y. Renard).
} 


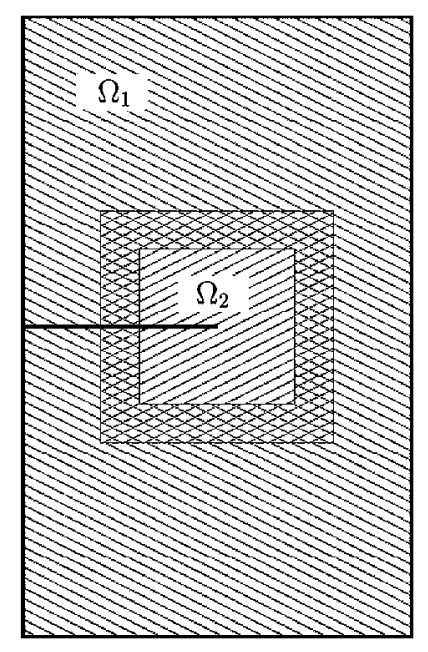

Fig. 1. Domain decomposition with PUFEM.

other elements totally or non-enriched. This issue was already addressed in many works by modifying the Xfem standard formulation or by introducing some approaches based on Xfem in order to overcome the blending elements drawback. Chessa et al. pointed out this issue from 2003 in [8] and they proposed two techniques to improve the convergence by modifying the construction of the blending elements in order to retrieve the partition of unity as much as possible. Using spectral elements together with the latter approach was carried out in [18]. In [13] the authors introduce a modified Xfem formulation that allows to the blending element to be totally enriched. This method improves the approximation error of standard Xfem. A coupling of XFEM with the hybrid crack element method was done also in [27] which allowed to avoid blending elements. Gracie et al. developed in [14] another technique based on a discontinuous Galerkin formulation using overlapping patches over the enriched areas. These patches localize the enrichment and they are bonded with the non-enriched area by penalizing the jump of the displacement and the stress field. Other works considered higher order blending elements which allows to enhance the approximation of standard XFEM [26].

A possibility to overcome the blending element issue is given also by the partition of unity method [20] applied to the elasticity problem on the cracked domain $\Omega$. Let us consider an overlapping of the finite element mesh over the uncracked domain $\bar{\Omega}$ by $\Omega_{1}$ and $\Omega_{2}$ such that the crack tip belongs to $\Omega_{2}$ (see Fig. 1). The finite element method defined on $\Omega_{1}$ (resp. on $\Omega_{2}$ ) is enriched by a step function near the crack sides (resp. by a step function near the crack sides and by some singular functions on the whole $\Omega_{2}$ ). The partition of unity defined by the $P_{1}$ finite element functions is used to keep the continuity of the displacement field through the transition layer $\Omega_{1} \cap \Omega_{2}$. A mathematical analysis in [17] proves that the width of the transition layer does not affect the quality of the approximation (see also [5]). Therefore the idea is to remove this layer by considering a partition $\Omega_{1}$ and $\Omega_{2}$ of the finite element mesh and to replace the transition by a nodal bonding condition at the interface between the disjoint subdomains $\Omega_{1}$ and $\Omega_{2}$ (see $[5,17]$ ).

In this paper, we study an approximation method that keeps the advantages of Xfem with a cut-off function and makes use of the previous idea. An mortar type integral matching condition (see [3]) is enforced on the interface between the singular enrichment surface $\Omega_{2}$ and the complementary part $\Omega_{1}$ of the finite element mesh. Let us point out that this interface coincides with element edges and does not cut any element into two parts. So, the proposed strategy differs from standard mortar approaches in the definition of the multiplier approximation space to approximate the exact multiplier discontinuous through the crack. In [17], the authors proposed a similar strategy using a pointwise matching condition. Numerical simulation showed optimal convergence results for this approach. The present work is devoted to the mathematical analysis of the new integral matching Xfem approach. An optimal error estimate will be obtained for this non-conformal approximation method. Some computational tests will be achieved to show the improved performances of the integral matching Xfem with respect to Xfem with enrichment surface and to Xfem with a cut-off function.

The outline of the paper is the following. Section 2 defines the finite element enriched spaces associated with the method. Section 3 presents the strong formulation of the elasticity problem defined over the partitioned domain, the associated hybrid weak formulation and an existence and uniqueness result. This weak formulation involves an integral matching condition on the interface between the two subdomains. In Section 4, we give a discrete formulation of the integral matching problem and prove a discrete inf-sup condition that will be used later. We also prove respectively in Sections 5, 6 and 7 the coercivity of the "broken" bilinear form in the hybrid formulation, an abstract error estimate as well as an approximation property for the multiplier space used to define the integral matching. A mathematical result of convergence for the integral matching Xfem method is given in Section 8. Finally, we perform some computational tests and comparisons with former Xfem methods in Section 9. 


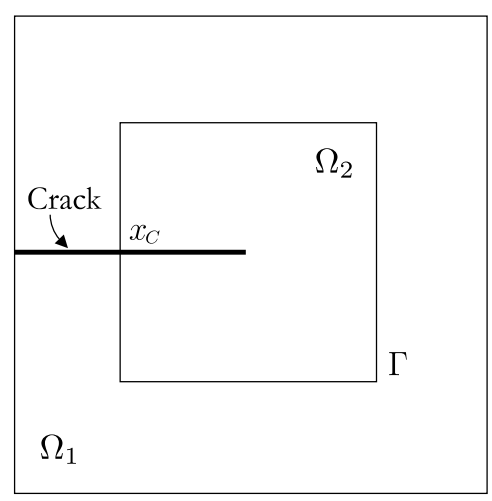

Fig. 2. Decomposition of the cracked domain.

\section{A non-conformal method}

Let $\Omega$ be a cracked bounded domain of $\mathbb{R}^{2}, \Gamma_{C}$ denotes the crack. For the sake of simplicity, $\Omega$ is assumed to be polygonal and $\Gamma_{C}$ a line segment. Let $\Omega_{1}$ and $\Omega_{2}$ be a partition of $\Omega$ such that the crack tip $x^{*}$ belongs to the interior of $\bar{\Omega}_{2}$ and the boundary $\partial \bar{\Omega}_{2}$ of $\bar{\Omega}_{2}$ is polygonal (see Fig. 2).

Let $\mathcal{T}_{h}$ be a regular family of triangulations (in the sense of Ciarlet [9]) defined on $\bar{\Omega}$ independently of the crack path such that $\partial \bar{\Omega}_{2}$ coincides with the edges of some elements of $\mathcal{T}_{h}, h$ being defined by

$$
h=\max _{T \in \mathcal{T}_{h}} \operatorname{diam}(T)=\max _{T \in \mathcal{T}_{h}}\left[\max _{x_{1}, x_{2} \in T}\left|x_{1}-x_{2}\right|\right] .
$$

We consider on $\mathcal{T}_{h}$ a $P_{1}$ finite element method with scalar shape functions denoted $\left\{\varphi_{i}\right\}_{i \in I}$. The basis function $\varphi_{i}$ is associated with the node $x_{i}$. Let $I_{H}$ be the set of indices corresponding to the shape functions whose support is completely cut by the crack. One of the Xfem enrichment consists of these shape functions multiplied by the Heaviside type function (see [21])

$$
H(x)= \begin{cases}+1 & \text { if }\left(x-x^{*}\right) \cdot n^{0} \geqslant 0 \\ -1 & \text { elsewhere }\end{cases}
$$

where $n^{0}$ is a given normal vector to the segment of line $\Gamma_{C}$. Let also $I\left(\Omega_{k}\right)$ (resp. $I_{H}\left(\Omega_{k}\right)$ ) be the subset of indices $i \in I$ (resp. $i \in I_{H}$ ) such that $x_{i} \in \bar{\Omega}_{k}, k \in\{1,2\}$.

Let $\mathcal{V}_{1}^{h}$ be the space of the classical $P_{1}$ finite element method on $\Omega_{1}$ enriched only by the discontinuity across the crack:

$$
\mathcal{V}_{1}^{h}=\left\{v_{1}^{h}: v_{1}^{h}=\sum_{i \in I\left(\Omega_{1}\right)} a_{i} \varphi_{\left.i\right|_{\Omega_{1}}}+\sum_{i \in I_{H}\left(\Omega_{1}\right)} b_{i} H \varphi_{\left.i\right|_{\Omega_{1}}} ; a_{i}, b_{i} \in \mathbb{R}^{2}\right\},
$$

and $\mathcal{V}_{2}^{h}$ the finite element space on $\Omega_{2}$ enriched by the discontinuity and by the crack tip functions:

$$
\mathcal{V}_{2}^{h}=\left\{v_{2}^{h}: v_{2}^{h}=\sum_{i \in I\left(\Omega_{2}\right)} a_{i} \varphi_{\left.i\right|_{\Omega_{2}}}+\sum_{i \in I_{H}\left(\Omega_{2}\right)} b_{i} H \varphi_{\left.i\right|_{\Omega_{2}}}+\sum_{j=1}^{4} c_{j} F_{\left.j\right|_{\Omega_{2}}} ; a_{i}, b_{i}, c_{j} \in \mathbb{R}^{2}\right\},
$$

where the singular enrichment functions are given by

$$
\left\{F_{j}(x)\right\}_{1 \leqslant j \leqslant 4}=\left\{\sqrt{r} \sin \frac{\theta}{2}, \sqrt{r} \cos \frac{\theta}{2}, \sqrt{r} \sin \frac{\theta}{2} \sin \theta, \sqrt{r} \cos \frac{\theta}{2} \sin \theta\right\},
$$

in the crack tip polar coordinates system (see [21]). Let us note that these functions are introduced in $\mathcal{V}_{2}^{h}$ globally over $\Omega_{2}$, which will add only eight additional lines to the final stiffness matrix.

In what follows, the approximation space of the displacement field on $\Omega$, denoted $\mathcal{V}^{h}$, is the set of functions $v^{h}$ defined on $\Omega$ such that $(k \in\{1,2\})$

$$
v^{h}=v_{k}^{h} \quad \text { in } \Omega_{k}, v_{k}^{h} \in \mathcal{V}_{k}^{h} .
$$

The space $\mathcal{V}^{h}$ can be identified to the product space $\mathcal{V}_{1}^{h} \times \mathcal{V}_{2}^{h}$. The following section defines a bonding condition for the functions $v^{h} \in \mathcal{V}^{h}$ to recover a weak continuity property across the interface between $\Omega_{1}$ and $\Omega_{2}$. 


\section{Hybrid formulation}

We recall the strong formulation of the elasticity problem on the cracked domain:

$$
\begin{aligned}
& \sigma=D \varepsilon(u) \quad \text { in } \Omega, \\
& -\operatorname{div} \sigma=g \quad \text { in } \Omega, \\
& u=0 \quad \text { on } \Gamma_{D}, \\
& \sigma n=f \text { on } \Gamma_{N}, \\
& \sigma n=0 \text { on } \Gamma_{C} .
\end{aligned}
$$

Here $u, \sigma$ and $\varepsilon(u)$ denote the displacement vector field, the stress tensor and the linearized strain tensor in plane elasticity, respectively; $D$ and div stand for the fourth order symmetric tensor of the elasticity coefficients and the divergence operator, $f$ and $g$ are given external loads, $n$ denotes the outward unit normal to $\Omega$ on $\partial \Gamma$ and finally $\Gamma_{D}$ and $\Gamma_{N}$ define a partition of the boundary of $\bar{\Omega}$ such that $\Gamma_{\mathrm{C}}$ and $\Gamma_{\mathrm{D}}$ are disjoint.

Note that considering a homogeneous Dirichlet boundary condition is not a restriction since the mathematical analysis can be straightforwardly extended to the non-homogeneous case. The non-penetrating condition on the crack sides is not taken into account. We suppose that

$$
\begin{aligned}
& g \in L^{2}\left(\Omega, \mathbb{R}^{2}\right), \quad f \in L^{2}\left(\Gamma_{N}, \mathbb{R}^{2}\right), \\
& \text { meas } \Gamma_{D}>0 .
\end{aligned}
$$

Taking into consideration the partition of the body $\Omega$ into $\Omega_{1}$ and $\Omega_{2}$, problem (7) can be written as a transmission problem in the space $\mathcal{V}$ of the discontinuous functions across the interface between $\Omega_{1}$ and $\Omega_{2}$, defined by

$$
\mathcal{V}=\left\{v \in L^{2}\left(\Omega, \mathbb{R}^{2}\right): v_{k}=\left.v\right|_{\Omega_{k}} \in H^{1}\left(\Omega_{k}, \mathbb{R}^{2}\right), v_{1}=0 \text { on } \Gamma_{D}\right\}
$$

(see [23]). Let $\mathcal{V}_{1}$ and $\mathcal{V}_{2}$ be the following spaces

$$
\mathcal{V}_{1}=\left\{v_{1} \in H^{1}\left(\Omega_{1}, \mathbb{R}^{2}\right): v_{1}=0 \text { on } \Gamma_{D}\right\},
$$

and

$$
\mathcal{V}_{2}=H^{1}\left(\Omega_{2}, \mathbb{R}^{2}\right)
$$

The space $\mathcal{V}$ can be identified to the space $\mathcal{V}_{1} \times \mathcal{V}_{2}$ equipped with the canonical norm of the product space $H^{1}\left(\Omega_{1}, \mathbb{R}^{2}\right) \times$ $H^{1}\left(\Omega_{2}, \mathbb{R}^{2}\right)$ :

$$
\|v\| \mathcal{V}=\left(\left\|v_{1}\right\|_{1, \Omega_{1}}^{2}+\left\|v_{2}\right\|_{1, \Omega_{2}}^{2}\right)^{1 / 2}
$$

where $v_{k}=\left.v\right|_{\Omega_{k}}$. Let $\Gamma$ be the interface between the two subdomains:

$$
\Gamma=\partial \bar{\Omega}_{2} \backslash\left\{x_{C}\right\},
$$

where $x_{C}$ is the intersection point of the boundary $\partial \bar{\Omega}_{2}$ and the crack $\Gamma_{C}$ (Fig. 2).

The jump of the displacement field through $\Gamma$ is denoted

$$
\llbracket u \rrbracket=\left.\left(u_{2}-u_{1}\right)\right|_{\Gamma} .
$$

We consider the following hybrid problem (see [4]):

Find $u \in \mathcal{V}, \lambda \in \mathcal{W}$ such that

$$
\begin{aligned}
& a(u, v)+b(v, \lambda)=L(v) \quad \forall v \in \mathcal{V}, \\
& b(u, \mu)=0 \quad \forall \mu \in \mathcal{W} .
\end{aligned}
$$

Denoting $u=\left(u_{1}, u_{2}\right)$, the "broken" bilinear form $a(\cdot, \cdot)$ is defined by

$$
a(u, v)=\sum_{k=1}^{2} a_{k}\left(u_{k}, v_{k}\right)=\sum_{k=1}^{2} \int_{\Omega_{k}} D \varepsilon\left(u_{k}\right): \varepsilon\left(v_{k}\right) d x,
$$

where $\sigma: \varepsilon=\sum_{i j} \sigma_{i j} \varepsilon_{i j}$. The operator $D$ of elasticity coefficients is assumed to satisfy a uniform ellipticity property (i.e. there exists $a_{0}>0$ such that $D \varepsilon: \varepsilon \geqslant a_{0} \varepsilon: \varepsilon$ for all symmetrical tensor $\varepsilon$, see [10]). Moreover, 


$$
L(v)=\sum_{k=1}^{2} \int_{\Omega_{k}} g \cdot v_{k} d x+\int_{\Gamma_{N}} f \cdot v_{1} d \Gamma,
$$

and

$$
b(v, \mu)=-\int_{\Gamma} \mu \cdot \llbracket v \rrbracket d \Gamma,
$$

where $u \cdot v=\sum_{i} u_{i} v_{i}$. The multiplier space $\mathcal{W}$ is the topological dual space $\left(H^{1 / 2}\left(\Gamma, \mathbb{R}^{2}\right)\right)^{\prime}=H_{00}^{-1 / 2}\left(\Gamma, \mathbb{R}^{2}\right)$ equipped with its canonical norm denoted $\left\|_{\cdot}\right\|_{-1 / 2, \Gamma}$. In Eq. (19), we still denote by an integral on $\Gamma$ the duality product between $H^{1 / 2}\left(\Gamma, \mathbb{R}^{2}\right)$ and its dual.

Lemma 3.1. Let us assume (8), (9). There exists a unique pair $(u, \lambda) \in \mathcal{V} \times \mathcal{W}$ solution to problem (16). Moreover, the displacement field $u$ is solution to the weak formulation associated with the elasticity problem (7).

Proof. For the sake of the self-consistency of the paper, the proof of this classical result is given. In fact, the existence and the uniqueness of the solution $(u, \lambda)$ to $(16)$ are obtained using a classical result for saddle point problems (see [4]). The main difficulty is to prove the coercivity of the bilinear form $a(\cdot, \cdot)$ on the kernel $\operatorname{Ker} B$ of the operator $B$. The latter is defined from $\mathcal{V}=\mathcal{V}_{1} \times \mathcal{V}_{2}$ into $H^{1 / 2}\left(\Gamma, \mathbb{R}^{2}\right)$ as

$$
\langle B v, \mu\rangle=b(v, \mu), \quad \forall \mu \in \mathcal{W},
$$

for all $v \in \mathcal{V}$. The elements of $\operatorname{Ker} B$ are the functions $v \in \mathcal{V}$ such that $\llbracket v \rrbracket=0$. Then the kernel of $B$ can be identified to the space

$$
V=\left\{v \in H^{1}\left(\Omega, \mathbb{R}^{2}\right): v=0 \text { on } \Gamma_{D}\right\},
$$

equipped with the $H^{1}\left(\Omega, \mathbb{R}^{2}\right)$-norm.

Using the definition (17) of $a(\cdot, \cdot)$ restricted to $V \times V$, we obtain

$$
a(v, v)=\int_{\Omega} D \varepsilon(v): \varepsilon(v) d x, \quad \forall v \in V .
$$

As a result of the Korn inequality (see [11]), there exists $\alpha>0$ such that

$$
a(v, v) \geqslant \alpha\|v\|_{\mathcal{V}}^{2}, \quad \forall v \in V .
$$

Consequently, the broken bilinear form $a(\cdot, \cdot)$ is coercive on $\operatorname{Ker} B$ and the hybrid problem (16) is well posed.

Now, Eq. (16b) means $u \in V$, then Eq. (16a) implies

$$
a(u, v)=L(v) \quad \forall v \in V,
$$

which means that $u$ is solution to the weak displacement elasticity problem on the cracked body $\Omega$.

Remark 1. At least in a weak sense, the multiplier $\lambda$ can be interpreted as being the normal stress to the interface $\Gamma$ (i.e. for $u$ sufficiently smooth one has $\lambda=\sigma(u) n$, where $n$ denotes the outward unit normal to $\Omega_{2}$ on $\Gamma$ ).

\section{Integral matching condition}

In this section, we write a discrete version of the hybrid problem considered in the previous section. We use the approximation space of discontinuous displacement fields on the interface $\Gamma$ introduced in Section 2 and we define a matching condition on $\Gamma$. We also prove an associated discrete inf-sup condition necessary for the convergence analysis.

We consider that the Dirichlet boundary $\Gamma_{D}$ is the union of some element edges of the triangulation $\mathcal{T}_{h}$. Moreover, in the definition of the approximation space $\mathcal{V}_{1}^{h}$ given by (3), the set $I\left(\Omega_{1}\right)$ does not contain the indices of the nodes that belong to $\Gamma_{D}$, in such a way that $\mathcal{V}^{h} \subset \mathcal{V}$.

The regular family of triangulations $\mathcal{T}_{h}$ introduced in Section 2 defines a regular family of subdivisions $\mathcal{S}_{h}$ of $\bar{\Gamma}$ into line segments, where $\bar{\Gamma}=\partial \bar{\Omega}_{2}$ (Figs. 2 and 3). We denote

$$
\mathcal{W}^{h}=\left\{\mu^{h} \in C^{0}(\bar{\Gamma})^{2}:\left.\mu_{i}^{h}\right|_{S} \in P_{1}, \forall S \in \mathcal{S}_{h}, i \in\{1,2\}\right\} .
$$

The set $\mathcal{W}^{h}$ can be identified to a subspace of $\mathcal{W}$ equipped with the induced norm. Approximation properties of such discrete multipliers will be examined in Section 7. 


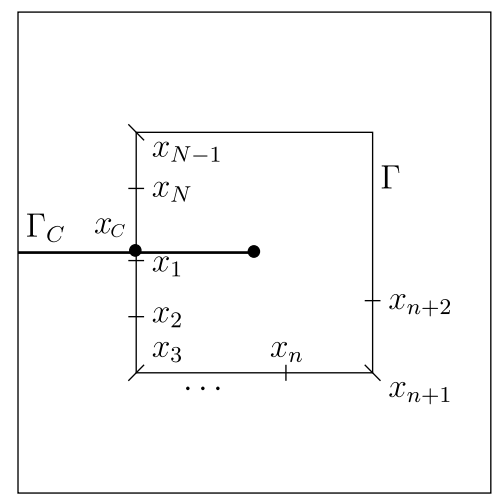

Fig. 3. Discretization of the interface $\Gamma$.

Then, the discrete formulation associated with the hybrid problem (16) can be written:

$$
\begin{aligned}
& \text { Find } u^{h} \in \mathcal{V}^{h}, \lambda^{h} \in \mathcal{W}^{h} \quad \text { such that } \\
& a\left(u^{h}, v^{h}\right)+b\left(v^{h}, \lambda^{h}\right)=L\left(v^{h}\right) \quad \forall v^{h} \in \mathcal{V}^{h}, \\
& b\left(u^{h}, \mu^{h}\right)=0 \quad \forall \mu^{h} \in \mathcal{W}^{h}
\end{aligned}
$$

where the displacement space $\mathcal{V}^{h}=\mathcal{V}_{1}^{h} \times \mathcal{V}_{2}^{h}$ is defined in Section 2.

In the enriched finite element space $\mathcal{V}^{h}$, the displacement fields are discontinuous through the interface $\Gamma$ : Eq. (26b) can be seen as an integral matching condition applied to the discrete solution $u^{h}$ on $\Gamma$.

Definition. Let $B^{h}$ be the operator defined from $\mathcal{V}^{h}$ into the dual of $\mathcal{W}^{h}$ such that, for all $v^{h} \in \mathcal{V}^{h}$ :

$$
\left\langle B^{h} v^{h}, \mu^{h}\right\rangle=b\left(v^{h}, \mu^{h}\right), \quad \forall \mu^{h} \in \mathcal{W}^{h}
$$

The following theorem gives a compatibility condition via the operator $B^{h}$ between the discrete displacement space $\mathcal{V}^{h}$ defined in Section 2 and the discrete multiplier space $\mathcal{W}^{h}$ defined by (25).

Theorem 4.1. The approximation spaces $\mathcal{V}^{h}$ and $\mathcal{W}^{h}$ satisfy:

$$
\inf _{\mu^{h} \in \mathcal{W}^{h}} \sup _{v^{h} \in \mathcal{V}^{h}} \frac{b\left(v^{h}, \mu^{h}\right)}{\left\|v^{h}\right\| \mathcal{V}\left\|\mu^{h}\right\|_{-1 / 2, \Gamma}} \geqslant \beta,
$$

where $\beta>0$ is a constant independent of $h$.

Proof. Let $X_{1}^{h}$ (resp. $X_{2}^{h}$ ) be the non-enriched finite element space defined on $\Omega_{1}$ (resp. $\Omega_{2}$ ). Precisely, $X_{1}^{h}$ (resp. $X_{2}^{h}$ ) is the set of continuous piecewise affine functions on $\left.\mathcal{T}_{h}\right|_{\Omega_{1}}$ (resp. on $\left.\mathcal{T}_{h}\right|_{\Omega_{2}}$ ) such that $v_{1}^{h}=0$ on $\Gamma_{D}$. The space $X_{k}^{h}$ is equipped with the $H^{1}\left(\Omega_{k}, \mathbb{R}^{2}\right)$-norm, $k \in\{1,2\}$.

Let $\mu^{h}$ be an element of $\mathcal{W}^{h}$. Using a result obtained in [2], there exists $v_{1}^{h} \in X_{1}^{h}$ such that

$$
\int_{\Gamma} \mu^{h} \cdot v_{1}^{h} d \Gamma \geqslant \beta_{1}\left\|\mu^{h}\right\|_{-1 / 2, \Gamma}\left\|v_{1}^{h}\right\|_{1, \Omega_{1}}
$$

where $\beta_{1}>0$ denotes a constant independent of $h, \mu^{h}$ and $v^{h}$. In the same way, there exists $-v_{2}^{h} \in X_{2}^{h}$ such that

$$
-\int_{\Gamma} \mu^{h} \cdot v_{2}^{h} d \Gamma \geqslant \beta_{2}\left\|\mu^{h}\right\|_{-1 / 2, \Gamma}\left\|v_{2}^{h}\right\|_{1, \Omega_{2}},
$$

where $\beta_{2}>0$ is a constant independent of $h$. Adding Eqs. (29) and (30) leads to

$$
-\int_{\Gamma} \mu^{h} \cdot \llbracket v^{h} \rrbracket d \Gamma \geqslant \beta\left\|\mu^{h}\right\|_{-1 / 2, \Gamma}\left(\left\|v_{1}^{h}\right\|_{1, \Omega_{1}}+\left\|v_{2}^{h}\right\|_{1, \Omega_{2}}\right),
$$

where $\beta=\inf \left(\beta_{1}, \beta_{2}\right)>0$. Consequently, 


$$
\begin{aligned}
b\left(v^{h}, \mu^{h}\right) & \geqslant \beta\left\|\mu^{h}\right\|_{-1 / 2, \Gamma}\left(\left\|v_{1}^{h}\right\|_{1, \Omega_{1}}^{2}+\left\|v_{2}^{h}\right\|_{1, \Omega_{2}}^{2}\right)^{1 / 2} \\
& \geqslant \beta\left\|\mu^{h}\right\|_{-1 / 2, \Gamma}\left\|v^{h}\right\|_{\mathcal{V}} .
\end{aligned}
$$

Since the enriched discrete space $\mathcal{V}^{h}$ contains $X_{1}^{h} \times X_{2}^{h}$, thus

$$
\sup _{v^{h} \in \mathcal{V}^{h}} \frac{b\left(v^{h}, \mu^{h}\right)}{\left\|v^{h}\right\| \mathcal{V}} \geqslant \beta\left\|\mu^{h}\right\|_{-1 / 2, \Gamma}, \quad \forall \mu^{h} \in \mathcal{W}^{h}
$$

\section{A coercivity property}

Unlike Eq. (16b), Eq. (26b) is not sufficient to have the continuity of the discrete displacement field through $\Gamma$. Then, the coercivity of the broken bilinear form $a(\cdot, \cdot)$ is not obvious in the finite element context. We now examine this difficulty.

Let us define the following subspace $V_{0}$ of discontinuous displacements by putting:

$$
\gamma=\int_{\Gamma} 1 d \Gamma, \quad \gamma_{1}=\int_{\Gamma} x_{1} d \Gamma, \quad \gamma_{2}=\int_{\Gamma} x_{2} d \Gamma, \quad \gamma_{3}=\int_{\Gamma}\left(x_{1}^{2}+x_{2}^{2}\right) d \Gamma,
$$

and

$$
\alpha_{1}=\gamma_{1} / \gamma, \quad \alpha_{2}=\gamma_{2} / \gamma
$$

We denote

$$
\mu_{1}(x)=(1,0), \quad \mu_{2}(x)=(0,1), \quad \mu_{3}(x)=\left(\alpha_{1}, x_{1}+\alpha_{2}\right), \quad \forall x \in \Gamma,
$$

and

$$
V_{0}=\left\{w \in \mathcal{V}: \int_{\Gamma} \mu_{i} \cdot w d \Gamma=0, i \in\{1,2,3\}\right\} .
$$

Lemma 5.1. Assuming (9), there exists $\alpha>0$ such that

$$
a(w, w) \geqslant \alpha\|w\|_{\mathcal{V}}^{2}, \quad \forall w \in V_{0} .
$$

Proof. The coercivity of $a(\cdot, \cdot)$ will be stated in two steps. The first one is to prove that the infinitesimal rigid displacements in $V_{0}$ are zero. The second one is to apply the Petree-Tartar lemma.

Let $w=\left(w_{1}, w_{2}\right)$ be a function of $V_{0}$ such that $(k \in\{1,2\})$

$$
\varepsilon\left(w_{k}\right)=0 \text { in } \Omega .
$$

In order to prove the first step, we have to show that

$$
w=0 \text { in } \Omega .
$$

Since $\mathcal{V}^{h} \subset \mathcal{V}$ and a Dirichlet condition is prescribed on a part $\Gamma_{D}$ of the boundary of $\Omega_{1}$ satisfying the condition (9), we obtain $w_{1}=0$ in $\Omega_{1}$. On the other hand, the condition $\varepsilon\left(w_{2}\right)=0$ can be written

$$
w_{2}=\left(a x_{2}+b_{1},-a x_{1}+b_{2}\right) .
$$

Now, since $w \in V_{0}$, it follows

$$
\left\{\begin{array}{l}
\gamma_{2} a+\gamma b_{1}=0, \\
-\gamma_{1} a+\gamma b_{2}=0, \\
\left(-\gamma_{3}+\alpha_{1} \gamma_{2}-\alpha_{2} \gamma_{1}\right) a+\left(-\gamma_{2}+\alpha_{1}\right) b_{1}+\left(\gamma_{1}+\alpha_{2}\right) b_{2}=0 .
\end{array}\right.
$$

Using the Schwarz inequality, we prove that the determinant of this system $\gamma\left(\gamma_{1}^{2}+\gamma_{2}^{2}-\gamma_{3} \gamma\right)$ is nonzero. Then the solution to system (42) is $\left(a, b_{1}, b_{2}\right)=(0,0,0)$. From (41), it results that $w_{2}=0$ in $\Omega_{2}$ and, consequently, assertion (40) holds.

Now, let $X=V_{0}$ be equipped with the $\mathcal{V}$-norm and

$$
\begin{aligned}
& Y=\left\{\varepsilon=\left(\varepsilon_{i j}\right), \varepsilon_{i j}=\left(\varepsilon_{i j}^{1}, \varepsilon_{i j}^{2}\right): \varepsilon_{i j}^{k} \in L^{2}\left(\Omega_{k}, \mathbb{R}\right), k \in\{1,2\}\right\}, \\
& Z=\left\{w=\left(w_{1}, w_{2}\right): w_{k} \in L^{2}\left(\Omega_{k}, \mathbb{R}^{2}\right), k \in\{1,2\}\right\},
\end{aligned}
$$

be equipped with the associated product norms. Let also $A$ be the mapping defined from $X$ into $Y$ such that 


$$
A(w)=\varepsilon(w)
$$

for every function $w \in X$ and $T$ the embedding operator from $X$ into $Z$. Using the Korn inequality, we obtain the existence of $\alpha_{k}>0$ such that

$$
\alpha_{k}\left\|w_{k}\right\|_{1, \Omega_{k}}^{2} \leqslant\left\|w_{k}\right\|_{0, \Omega_{k}}^{2}+\|\varepsilon(v)\|_{0, \Omega_{k}}^{2}, \quad \forall w_{k} \in H^{1}\left(\Omega_{k}, \mathbb{R}^{2}\right),
$$

i.e. $\left(c=\inf \left\{\alpha_{1}, \alpha_{2}\right\}\right)$

$$
c\|w\|_{X}^{2} \leqslant\|T(w)\|_{Z}^{2}+\|A(w)\|_{Y}^{2}, \quad \forall w \in X
$$

Moreover, the operator $A$ is injective due to the first step of the proof. Finally, the operator $T$ is compact due to the compactness of the embedding operator from $H^{1}(\Omega)$ into $L^{2}(\Omega)$. Now, the Petree-Tartar lemma (see [12] for instance) implies the existence of $\alpha>0$ satisfying

$$
\alpha\|w\|_{X}^{2} \leqslant\|A(w)\|_{Y}^{2}, \quad \forall w \in X
$$

Using the ellipticity property of the elasticity coefficients (Section 3 ), the latter result leads to the coercivity of $a(\cdot, \cdot)$ in $V_{0}$.

Corollary 5.2. There exists a unique pair $\left(u^{h}, \lambda^{h}\right) \in \mathcal{V}^{h} \times \mathcal{W}^{h}$ solution to problem (26).

Proof. In order to follow the proof of Lemma 3.1, it is sufficient to have the coercivity of the bilinear form $a(\cdot, \cdot)$ on Ker $B^{h}$. Since $\operatorname{Ker} B^{h} \subset V_{0}$, the broken bilinear form $a(\cdot, \cdot)$ is coercive on Ker $B^{h}$ (Lemma 5.1). Then, the existence and the uniqueness of a solution $\left(u^{h}, \lambda^{h}\right)$ to the saddle point problem $(26)$ are obtained as for the continuous weak formulation.

Remark 2. The continuity through the interface $\Gamma$ of the discrete displacement field is prescribed in $\mathcal{V}^{h}$ only in a weak sense given by the integral matching condition (26b). Consequently, the discrete space $\mathcal{V}^{h}$ cannot be identified to a subspace of $H^{1}\left(\Omega, \mathbb{R}^{2}\right)$ and the discrete hybrid problem (26) corresponds to a non-conformal approximation method. Another type of non-conformal approximation is defined by the nodal matching condition (see [17]):

$$
\llbracket u \rrbracket=0 \text { at every node on } \Gamma \text {. }
$$

\section{Abstract error estimate}

The discrete inf-sup condition (28) and the coercivity property for the broken bilinear form $a(\cdot, \cdot)$ allow to obtain an abstract error estimate associated with the Xfem with integral matching condition.

Proposition 6.1. Let $(u, \lambda)$ and $\left(u^{h}, \lambda^{h}\right)$ be the solutions to problem (16) and problem (26), respectively. We have

$$
\left\|u-u^{h}\right\|_{\mathcal{V}}^{2}+\left\|\lambda-\lambda^{h}\right\|_{-1 / 2, \Gamma}^{2} \leqslant C\left\{\inf _{v^{h} \in \mathcal{V}^{h}}\left\|u-v^{h}\right\|_{\mathcal{V}}^{2}+\inf _{\mu^{h} \in \mathcal{W}^{h}}\left\|\lambda-\mu^{h}\right\|_{-1 / 2, \Gamma}^{2}\right\},
$$

where $C$ denotes a constant independent of $h$.

Proof. The proof of this proposition consists in finding successively an estimate of $\left\|u-u^{h}\right\|_{\mathcal{V}}$ and $\left\|\lambda-\lambda^{h}\right\|_{-1 / 2, \Gamma}$. The operators $B$ and $B^{h}$ introduced in (20) and (27) respectively satisfy the inclusion

$$
\operatorname{Ker} B+\operatorname{Ker} B^{h} \subset V_{0} \text {, }
$$

where the subspace $V_{0}$ is defined in (37). So the bilinear form $a(\cdot, \cdot)$ is coercive on $\operatorname{Ker} B+\operatorname{Ker} B^{h}$ (Lemma 5.1 ), then there exists $\alpha>0$ such that

$$
\alpha\left\|u-u^{h}\right\|_{\mathcal{V}}^{2} \leqslant a\left(u-u^{h}, u-u^{h}\right) .
$$

Using the bilinearity of $a(\cdot, \cdot)$, we obtain

$$
\alpha\left\|u-u^{h}\right\|_{\mathcal{V}}^{2} \leqslant a\left(u-u^{h}, u-v^{h}\right)+a\left(u, v^{h}-u^{h}\right)-a\left(u^{h}, v^{h}-u^{h}\right),
$$

for all $v^{h}$ in $\mathcal{V}^{h}$. Since $\mathcal{V}^{h} \subset \mathcal{V}$, Eqs. (16a) and (26a) in conjunction with (53) imply:

$$
\begin{aligned}
\alpha\left\|u-u^{h}\right\|_{\mathcal{V}}^{2} & \leqslant a\left(u-u^{h}, u-v^{h}\right)-b\left(v^{h}-u^{h}, \lambda\right)+b\left(v^{h}-u^{h}, \lambda^{h}\right) \\
& \leqslant a\left(u-u^{h}, u-v^{h}\right)-b\left(v^{h}-u, \lambda-\lambda^{h}\right)-b\left(u-u^{h}, \lambda-\lambda^{h}\right),
\end{aligned}
$$


because of the bilinearity of $b(\cdot, \cdot)$. Since $\mathcal{W}^{h} \subset \mathcal{W}$, Eqs. (16b) and (26b) lead to

$$
b\left(u-u^{h}, \mu^{h}-\lambda^{h}\right)=0,
$$

thus, for all $\mu^{h} \in \mathcal{W}^{h}$,

$$
b\left(u-u^{h}, \lambda-\lambda^{h}\right)=b\left(u-u^{h}, \lambda-\mu^{h}\right) .
$$

Consequently, we have

$$
\alpha\left\|u-u^{h}\right\|_{\mathcal{V}}^{2} \leqslant a\left(u-u^{h}, u-v^{h}\right)-b\left(v^{h}-u, \lambda-\lambda^{h}\right)-b\left(u-u^{h}, \lambda-\mu^{h}\right) .
$$

Now, denoting $C_{a}$ (resp. $C_{b}$ ) the continuity constant of the bilinear form $a(\cdot, \cdot)($ resp. $b(\cdot, \cdot))$, we have

$$
\begin{aligned}
\alpha\left\|u-u^{h}\right\|_{\mathcal{V}}^{2} \leqslant & C_{a}\left(\left\|u-u^{h}\right\|_{\mathcal{V}}\left\|u-v^{h}\right\|_{\mathcal{V}}\right)+C_{b}\left(\left\|u-v^{h}\right\|_{\mathcal{V}}\left\|\lambda-\lambda^{h}\right\|_{-1 / 2, \Gamma}\right. \\
& \left.+\left\|u-u^{h}\right\|_{\mathcal{V}}\left\|\lambda-\mu^{h}\right\|_{-1 / 2, \Gamma}\right) .
\end{aligned}
$$

Then

$$
\begin{aligned}
\left\|u-u^{h}\right\|_{\mathcal{V}}^{2} \leqslant & C\left\{\left(\left\|u-u^{h}\right\|_{\mathcal{V}}+\left\|\lambda-\lambda^{h}\right\|_{-1 / 2, \Gamma}\right) \inf _{v^{h} \in \mathcal{V}^{h}}\left\|u-v^{h}\right\|_{\mathcal{V}}\right. \\
& \left.+\left\|u-u^{h}\right\|_{\mathcal{V}} \inf _{\mu^{h} \in \mathcal{W}^{h}}\left\|\lambda-\mu^{h}\right\|_{-1 / 2, \Gamma}\right\},
\end{aligned}
$$

where $C$ is a generic constant independent of $h$.

An estimate of $\left\|\lambda-\lambda^{h}\right\|_{-1 / 2, \Gamma}$ is now obtained from the discrete inf-sup condition (28) which reads

$$
\begin{aligned}
\beta\left\|\lambda^{h}-\mu^{h}\right\|_{-1 / 2, \Gamma} & \leqslant \sup _{v^{h} \in \mathcal{V}^{h}} \frac{b\left(v^{h}, \lambda^{h}-\mu^{h}\right)}{\left\|v^{h}\right\| \mathcal{V}} \\
& \leqslant \sup _{v^{h} \in \mathcal{V}^{h}}\left(\frac{b\left(v^{h}, \lambda^{h}-\lambda\right)+b\left(v^{h}, \lambda-\mu^{h}\right)}{\left\|v^{h}\right\| \mathcal{V}}\right),
\end{aligned}
$$

for all $\mu^{h} \in \mathcal{W}^{h}$. Eqs. (16a) and (26a) lead to

$$
\begin{aligned}
\beta\left\|\lambda^{h}-\mu^{h}\right\|_{-1 / 2, \Gamma} & \leqslant \sup _{v^{h} \in \mathcal{V}^{h}}\left(\frac{a\left(u-u^{h}, v^{h}\right)+b\left(v^{h}, \lambda-\mu^{h}\right)}{\left\|v^{h}\right\|_{\mathcal{V}}}\right) \\
& \leqslant C_{a}\left\|u-u^{h}\right\|_{\mathcal{V}}+C_{b}\left\|\lambda-\mu^{h}\right\|_{-1 / 2, \Gamma^{\prime}}
\end{aligned}
$$

Using this latter inequality, one can write

$$
\begin{aligned}
\left\|\lambda-\lambda^{h}\right\|_{-1 / 2, \Gamma} & \leqslant\left\|\lambda-\mu^{h}\right\|_{-1 / 2, \Gamma}+\left\|\mu^{h}-\lambda^{h}\right\|_{-1 / 2, \Gamma} \\
& \leqslant \frac{1}{\beta}\left(C_{a}\left\|u-u^{h}\right\|_{\mathcal{V}}+\left(C_{b}+\beta\right)\left\|\lambda-\mu^{h}\right\|_{-1 / 2, \Gamma}\right),
\end{aligned}
$$

then

$$
\left\|\lambda-\lambda^{h}\right\|_{-1 / 2, \Gamma} \leqslant C\left(\left\|u-u^{h}\right\|_{\mathcal{V}}+\inf _{\mu^{h} \in \mathcal{W}^{h}}\left\|\lambda-\mu^{h}\right\|_{-1 / 2, \Gamma}\right) .
$$

Finally, from estimate (58), we get

$$
\begin{aligned}
\left\|u-u^{h}\right\|_{\mathcal{V}}^{2} \leqslant & C\left(\left\|u-u^{h}\right\|_{\mathcal{V}}+\inf _{\mu^{h} \in \mathcal{W}^{h}}\left\|\lambda-\mu^{h}\right\|_{-1 / 2, \Gamma}\right) \inf _{v^{h} \in \mathcal{V}^{h}}\left\|u-v^{h}\right\|_{\mathcal{V}} \\
& +\left\|u-u^{h}\right\|_{\mathcal{V}} \inf _{\mu^{h} \in \mathcal{W}^{h}}\left\|\lambda-\mu^{h}\right\|_{-1 / 2, \Gamma} .
\end{aligned}
$$

Then

$$
\left\|u-u^{h}\right\|_{\mathcal{V}}^{2} \leqslant C\left\{\inf _{v^{h} \in \mathcal{V}^{h}}\left\|u-v^{h}\right\|_{\mathcal{V}}^{2}+\inf _{\mu^{h} \in \mathcal{W}^{h}}\left\|\lambda-\mu^{h}\right\|_{-1 / 2, \Gamma}^{2}\right\} .
$$

Combining (61) and (64), we prove the proposition.

In order to exploit the latter error estimate, the next section is dedicated to the approximation of the exact multiplier. 


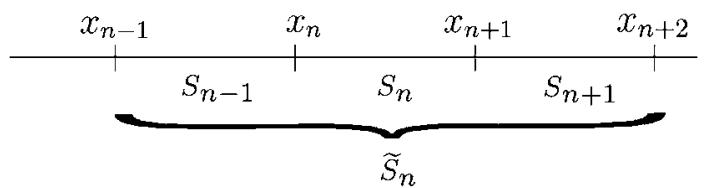

Fig. 4. The set of line segments $S_{n}$.

\section{Approximation property on multipliers}

The aim of this section is to obtain an approximation property on the exact multiplier space, i.e. the dual of $H^{1 / 2}\left(\Gamma, \mathbb{R}^{2}\right)$, where $\Gamma=\bar{\Gamma} \backslash\left\{x_{C}\right\}$.

Let $x_{n}, n=1, \ldots, N$, be the ordered nodes of the triangulation $\mathcal{T}_{h}$ which belong to $\bar{\Gamma}$. The set of line segments

$$
S_{n}=\left[x_{n}, x_{n+1}[, \quad n=1, \ldots, N,\right.
$$

where $x_{N+1}=x_{1}$, defines a partition of $\bar{\Gamma}$. The crack $\Gamma_{C}$ cuts the line segment $S_{N}$ (Fig. 3). We also denote $x_{N+2}=x_{2}$ and $x_{0}=x_{N}$.

In this section, let $Y^{h}$ be the discrete space of scalar functions defined by

$$
Y^{h}=\left\{\varphi^{h} \in C^{0}(\bar{\Gamma}):\left.\varphi^{h}\right|_{S_{n}} \in P_{1}, n=1, \ldots, N\right\} .
$$

The space $Y^{h}$ can be identified to a subset of $L^{2}(\Gamma, \mathbb{R})$. The following better approximation property holds.

Proposition 7.1. Let $\varphi \in H^{1 / 2}(\Gamma, \mathbb{R})$, then we have

$$
\inf _{\varphi^{h} \in Y^{h}}\left\|\varphi-\varphi^{h}\right\|_{-1 / 2, \Gamma} \leqslant C h\|\varphi\|_{1 / 2, \Gamma},
$$

where $C$ is a generic constant independent of $h$.

The proof of this proposition is based on the definition of the following Chen-Nochetto type approximation operator (see [7]). A similar construction can be found in [12] and [25]. Note also that an analogous approximation result was obtained in [16] for a different type of problem with signed multipliers.

Definition 7.2. Let $\varphi \in L^{1}(\Gamma, \mathbb{R})$, then $\Pi^{h} \varphi$ is the element of $Y^{h}$ such that

$$
\Pi^{h} \varphi\left(x_{n}\right)=\frac{1}{\left|S_{n-1} \cup S_{n}\right|} \int_{S_{n-1} \cup S_{n}} \varphi d \Gamma, \quad n=1, \ldots, N,
$$

where $|S|=\operatorname{meas}(S)$ (Fig. 4 ).

Let us first prove some intermediary results.

Lemma 7.3. Let $\varphi \in L^{1}(\Gamma, \mathbb{R})$, then $\Pi^{h} \varphi$ satisfies

$$
\left\|\Pi^{h} \varphi\right\|_{0, S_{n}} \leqslant 2\|\varphi\|_{0, \tilde{S}_{n}},
$$

where $\tilde{S}_{n}=S_{n-1} \cup S_{n} \cup S_{n+1}, n=1, \ldots, N+1$ (Fig. 4).

Proof. Let $\psi_{n}$ be the $P_{1}$ finite element basis function in $Y^{h}$ associated with the node $x_{n}$. Then

$$
\begin{aligned}
\left\|\Pi^{h} \varphi\right\|_{0, S_{n}} & =\left\|\Pi^{h} \varphi\left(x_{n}\right) \psi_{n}+\Pi^{h} \varphi\left(x_{n+1}\right) \psi_{n+1}\right\|_{0, S_{n}} \\
& \leqslant\left|\Pi^{h} \varphi\left(x_{n}\right)\right|\left\|\psi_{n}\right\|_{0, S_{n}}+\left|\Pi^{h} \varphi\left(x_{n+1}\right)\right|\left\|\psi_{n+1}\right\|_{0, S_{n}} .
\end{aligned}
$$

Since $\left|\psi_{n}(x)\right| \leqslant 1$, we have

$$
\left\|\psi_{n}\right\|_{0, S_{n}} \leqslant\left\{\operatorname{meas}\left(S_{n}\right)\right\}^{1 / 2} \leqslant h^{1 / 2},
$$

then

$$
\left\|\Pi^{h} \varphi\right\|_{0, S_{n}} \leqslant h^{1 / 2}\left(\left|\Pi^{h} \varphi\left(x_{n}\right)\right|+\left|\Pi^{h} \varphi\left(x_{n+1}\right)\right|\right) .
$$


Using the definition of $\Pi^{h} \varphi$, we obtain

$$
\begin{aligned}
\left\|\Pi^{h} \varphi\right\|_{0, S_{n}}^{2} & \leqslant 2 h\left(\left|\Pi^{h} \varphi\left(x_{n}\right)\right|^{2}+\left|\Pi^{h} \varphi\left(x_{n+1}\right)\right|^{2}\right) \\
& \leqslant \frac{1}{2 h}\left\{\left(\int_{x_{n-1}}^{x_{n+1}} \varphi d x\right)^{2}+\left(\int_{x_{n}}^{x_{n+2}} \varphi d x\right)^{2}\right\} .
\end{aligned}
$$

The Cauchy-Schwarz inequality leads to

$$
\begin{aligned}
\left\|\Pi^{h} \varphi\right\|_{0, S_{n}}^{2} & \leqslant \int_{x_{n-1}}^{x_{n+1}} \varphi^{2} d x+\int_{x_{n}}^{x_{n+2}} \varphi^{2} d x \\
& \leqslant 2\|\varphi\|_{0, \tilde{S}_{n}}^{2} .
\end{aligned}
$$

Lemma 7.4. Let $\varphi \in H^{1 / 2}(\Gamma, \mathbb{R})$. The following estimate holds

$$
\left\|\varphi-\Pi^{h} \varphi\right\|_{0, \Gamma} \leqslant C h^{1 / 2}\|\varphi\|_{1 / 2, \Gamma}
$$

where $C$ is a generic constant independent of $h$ and $\varphi$.

Proof. In order to prove the estimate over $\Gamma$, we proceed first by computing the local approximation errors $\left\|\varphi-\Pi^{h} \varphi\right\|_{0, S_{n}}$, for all $n$.

Let $\psi \in H^{1 / 2}(\Gamma, \mathbb{R})$ such that $\left.\psi\right|_{\tilde{S}_{n}}=c$, where $c$ is a constant. By using the definition of $\Pi^{h}$, we have

$$
\Pi^{h} \psi\left(x_{n}\right)=c=\Pi^{h} \psi\left(x_{n+1}\right),
$$

thus

$$
\left.\Pi^{h} \psi\right|_{s_{n}}=c .
$$

Since $\Pi^{h}$ is a linear operator, it follows

$$
\varphi-\Pi^{h} \varphi=(\varphi-c)-\Pi^{h}(\varphi-c) \text { on } S_{n},
$$

then

$$
\left\|\varphi-\Pi^{h} \varphi\right\|_{0, S_{n}} \leqslant\|\varphi-c\|_{0, S_{n}}+\left\|\Pi^{h}(\varphi-c)\right\|_{0, S_{n}} .
$$

Using Lemma 7.3 together with the latter inequality, we obtain

$$
\begin{aligned}
\left\|\varphi-\Pi^{h} \varphi\right\|_{0, S_{n}} & \leqslant\|\varphi-c\|_{0, S_{n}}+2\|\varphi-c\|_{0, \tilde{S}_{n}} \\
& \leqslant 3\|\varphi-c\|_{0, \tilde{S}_{n}} .
\end{aligned}
$$

Let

$$
c=\frac{1}{\left|\tilde{S}_{n}\right|} \int_{\tilde{S}_{n}} \varphi(y) d y,
$$

thus, for almost every $x \in \tilde{S}_{n}$,

$$
\varphi(x)-c=\frac{1}{\left|\tilde{S}_{n}\right|} \int_{\tilde{S}_{n}}(\varphi(x)-\varphi(y)) d y .
$$

For $2 \leqslant n \leqslant N-2$, the point $x_{C} \notin \tilde{S}_{n}$, then we have

$$
\varphi(x)-c=\frac{1}{\left|\tilde{S}_{n}\right|} \int_{\tilde{S}_{n}} \frac{\varphi(x)-\varphi(y)}{|x-y|}|x-y| d y,
$$

and the Cauchy-Schwarz inequality leads to 


$$
\begin{aligned}
\int_{\tilde{S}_{n}}|\varphi(x)-c|^{2} d x & \leqslant \frac{1}{\left|\tilde{S}_{n}\right|^{2}} \int_{\tilde{S}_{n}}\left\{\int_{\tilde{S}_{n}} \frac{|\varphi(x)-\varphi(y)|^{2}}{|x-y|^{2}} d y \int_{\tilde{S}_{n}}|x-y|^{2} d y\right\} d x \\
& \leqslant\left|\tilde{S}_{n}\right| \int_{\tilde{S}_{n}} \int_{\Gamma} \frac{|\varphi(x)-\varphi(y)|^{2}}{|x-y|^{2}} d x d y .
\end{aligned}
$$

Thus, using (80), we obtain

$$
\left\|\varphi-\Pi^{h} \varphi\right\|_{0, S_{n}}^{2} \leqslant 27 h \int_{\tilde{S}_{n}} \int_{\Gamma} \frac{|\varphi(x)-\varphi(y)|^{2}}{|x-y|^{2}} d x d y .
$$

For $n \in\{1, N-1, N\}$, we have $x_{C} \in \tilde{S}_{n}$. Denoting

$$
\tilde{S}_{n}^{-}=\left\{x \in \tilde{S}_{n}, x<x_{C}\right\} \text { and } \tilde{S}_{n}^{+}=\left\{x \in \tilde{S}_{n}, x>x_{C}\right\},
$$

Eq. (82) can be written

$$
\varphi(x)-c=\frac{1}{\left|\tilde{S}_{n}\right|}\left\{\int_{\tilde{S}_{n}^{+}}(\varphi(x)-\varphi(y)) d y+\int_{\tilde{S}_{n}^{-}}(\varphi(x)-\varphi(y)) d y\right\} .
$$

Then

$$
|\varphi(x)-c|^{2} \leqslant \frac{2}{\left|\tilde{S}_{n}\right|^{2}}\left\{\left(\int_{\tilde{S}_{n}^{+}}(\varphi(x)-\varphi(y)) d y\right)^{2}+\left(\int_{\tilde{S}_{n}^{-}}(\varphi(x)-\varphi(y)) d y\right)^{2}\right\} .
$$

Now, as in Eq. (84), the Cauchy-Schwarz inequality applied separately for the integrals on $\tilde{S}_{n}^{+}$and $\tilde{S}_{n}^{-}$leads to

$$
\begin{aligned}
|\varphi(x)-c|^{2} & \leqslant \frac{2}{\left|\tilde{S}_{n}\right|^{2}}\left\{\left|\tilde{S}_{n}^{+}\right|^{3}+\left|\tilde{S}_{n}^{-}\right|^{3}\right\} \int_{\Gamma} \frac{|\varphi(x)-\varphi(y)|^{2}}{|x-y|^{2}} d y \\
& \leqslant 4\left|\tilde{S}_{n}\right| \int_{\Gamma} \frac{|\varphi(x)-\varphi(y)|^{2}}{|x-y|^{2}} d y .
\end{aligned}
$$

By integrating on $\tilde{S}_{n}$, we have

$$
\begin{aligned}
& \int_{\tilde{S}_{n}}|\varphi(x)-c|^{2} d x \leqslant 4\left|\tilde{S}_{n}\right|\left\{\int_{\tilde{S}_{n}^{+}} \int_{\Gamma} \frac{|\varphi(x)-\varphi(y)|^{2}}{|x-y|^{2}} d x d y\right. \\
&\left.+\int_{\tilde{S}_{n}^{-}} \int_{\Gamma} \frac{|\varphi(x)-\varphi(y)|^{2}}{|x-y|^{2}} d x d y\right\} \\
& \leqslant 4\left|\tilde{S}_{n}\right| \int_{\Gamma} \int_{\Gamma} \frac{|\varphi(x)-\varphi(y)|^{2}}{|x-y|^{2}} d x d y .
\end{aligned}
$$

Consequently, using (80), we get

$$
\left\|\varphi-\Pi^{h} \varphi\right\|_{0, S_{n}}^{2} \leqslant 108 h\|\varphi\|_{1 / 2, \Gamma}^{2} .
$$

Finally, the global approximation error is obtained by summing the local errors (Eqs. (85) and (91)) as follows

$$
\begin{aligned}
\left\|\varphi-\Pi^{h} \varphi\right\|_{0, \Gamma}^{2} & =\sum_{n=2, \ldots, N-2}\left\|\varphi-\Pi^{h} \varphi\right\|_{0, S_{n}}^{2}+\sum_{n \in\{1, N-1, N\}}\left\|\varphi-\Pi^{h} \varphi\right\|_{0, S_{n}}^{2} \\
& \leqslant C h\|\varphi\|_{1 / 2, \Gamma}^{2} .
\end{aligned}
$$


Proof of Proposition 7.1. Let us define the $L^{2}$-projection operator $P^{h}$ onto $Y^{h}$, i.e. for all $\varphi \in L^{2}(\Gamma, \mathbb{R})$ :

$$
P^{h} \varphi \in Y^{h} \text { and } \int_{\Gamma}\left(\varphi-P^{h} \varphi\right) \cdot \psi^{h} d \Gamma=0, \quad \forall \psi^{h} \in Y^{h} .
$$

For every $\varphi \in H^{1 / 2}(\Gamma, \mathbb{R})$, we have

$$
\left\|\varphi-P^{h} \varphi\right\|_{-1 / 2, \Gamma}=\sup _{\psi \in H^{1 / 2}(\Gamma, \mathbb{R})} \frac{\left\langle\varphi-P^{h} \varphi, \psi\right\rangle_{-1 / 2,1 / 2, \Gamma}}{\|\psi\|_{1 / 2, \Gamma}},
$$

where $\|\psi\|_{1 / 2, \Gamma} \neq 0$. Since $\varphi-P^{h} \varphi$ belongs to the orthogonal to $Y^{h}$, then

$$
\left\|\varphi-P^{h} \varphi\right\|_{-1 / 2, \Gamma}=\sup _{\psi \in H^{1 / 2}(\Gamma, \mathbb{R})} \frac{\int_{\Gamma}\left(\varphi-P^{h} \varphi\right)\left(\psi-P^{h} \psi\right) d \Gamma}{\|\psi\|_{1 / 2, \Gamma}} .
$$

Using the Cauchy-Schwarz inequality

$$
\left\|\varphi-P^{h} \varphi\right\|_{-1 / 2, \Gamma} \leqslant\left\|\varphi-P^{h} \varphi\right\|_{0, \Gamma} \sup _{\psi \in H^{1 / 2}(\Gamma, \mathbb{R})} \frac{\left\|\psi-P^{h} \psi\right\|_{0, \Gamma}}{\|\psi\|_{1 / 2, \Gamma}} .
$$

Moreover, the element $P^{h} \psi$ satisfies, for all $\psi^{h} \in Y^{h}$

$$
\left\|\psi-P^{h} \psi\right\|_{0, \Gamma} \leqslant\left\|\psi-\psi^{h}\right\|_{0, \Gamma},
$$

so we derive from (96) that

$$
\left\|\varphi-P^{h} \varphi\right\|_{-1 / 2, \Gamma} \leqslant\left\|\varphi-\Pi^{h} \varphi\right\|_{0, \Gamma} \sup _{\psi \in H^{1 / 2}(\Gamma, \mathbb{R})} \frac{\left\|\psi-\Pi^{h} \psi\right\|_{0, \Gamma}}{\|\psi\|_{1 / 2, \Gamma}} .
$$

Since $P^{h} \varphi$ belongs to $Y^{h}$, the better approximation error is bounded as follows

$$
\inf _{\varphi^{h} \in Y^{h}}\left\|\varphi-\varphi^{h}\right\|_{-1 / 2, \Gamma} \leqslant\left\|\varphi-P^{h} \varphi\right\|_{-1 / 2, \Gamma} .
$$

Therefore, the estimate (98) reads

$$
\inf _{\varphi^{h} \in Y^{h}}\left\|\varphi-\varphi^{h}\right\|_{-1 / 2, \Gamma} \leqslant\left\|\varphi-\Pi^{h} \varphi\right\|_{0, \Gamma} \sup _{\psi \in H^{1 / 2}(\Gamma, \mathbb{R})} \frac{\left\|\psi-\Pi^{h} \psi\right\|_{0, \Gamma}}{\|\psi\|_{1 / 2, \Gamma}} .
$$

Finally, applying Lemma 7.4 leads to

$$
\inf _{\varphi^{h} \in Y^{h}}\left\|\varphi-\varphi^{h}\right\|_{-1 / 2, \Gamma} \leqslant C h\|\varphi\|_{1 / 2, \Gamma}
$$

Let us now apply Proposition 7.1 to the discrete multiplier space $\mathcal{W}^{h}=Y^{h} \times Y^{h}$ in the formulation of the fracture problem. Let $\mu \in H^{1 / 2}\left(\Gamma, \mathbb{R}^{2}\right)$, then we have

$$
\inf _{\mu^{h} \in \mathcal{W}^{h}}\left\|\mu-\mu^{h}\right\|_{-1 / 2, \Gamma} \leqslant C h\|\mu\|_{1 / 2, \Gamma} .
$$

Remark 3. In particular, this latter result implies that the representation of the discontinuity across the crack in the multiplier discrete space is not mandatory to obtain an optimal convergence rate.

\section{Convergence error}

This section presents the convergence result for Xfem with the integral matching condition on $\Gamma$. It is obtained from the abstract error estimate (Section 6), the approximation property in the multiplier space (Section 7 ) and the analysis developed in [6,22] on interpolation operators adapted to Xfem.

Let us recall that the displacement solution of the weak formulation of the fracture problem can be decomposed into $u=u_{r}+u_{s}$ where $u_{r}$ is a "regular" field over the cracked domain $\Omega$ and $u_{s}$ is the asymptotic displacement at the crack tip which is a linear combination of the two following fracture modes given in polar coordinates relatively to the crack tip $(E, v$ are the Young modulus and Poisson ratio, respectively, see $[19,15])$ : 


$$
\begin{aligned}
& u_{I}(r, \theta)=\frac{1}{E} \sqrt{\frac{r}{2 \pi}}(1+v)\left(\begin{array}{c}
\cos \frac{\theta}{2}(\delta-\cos \theta) \\
\sin \frac{\theta}{2}(\delta-\cos \theta)
\end{array}\right), \\
& u_{I I}(r, \theta)=\frac{1}{E} \sqrt{\frac{r}{2 \pi}}(1+v)\left(\begin{array}{c}
\sin \frac{\theta}{2}(\delta+2+\cos \theta) \\
\cos \frac{\theta}{2}(\delta-2+\cos \theta)
\end{array}\right) .
\end{aligned}
$$

Note that these two modes are themselves linear combinations of the singular enrichment functions $F_{j}$ introduced in Section 2.

Theorem 8.1. Let $(u, \lambda)$ be the solution to problem (16). Suppose that

$$
\lambda \in H^{1 / 2}\left(\Gamma, \mathbb{R}^{2}\right),
$$

and there exists $u_{s}$, a linear combination of $u_{I}$ and $u_{I I}$, such that $u_{r}=u-u_{s}$ satisfies

$$
u_{r} \in H^{2}\left(\Omega, \mathbb{R}^{2}\right) .
$$

Then the solution $\left(u^{h}, \lambda^{h}\right)$ to problem (26) satisfies

$$
\left\|u-u^{h}\right\|_{\mathcal{V}}+\left\|\lambda-\lambda^{h}\right\|_{-1 / 2, \Gamma} \leqslant \operatorname{Ch}\left(\|u\|_{2, \Omega_{1}}+\left\|u-u_{s}\right\|_{2, \Omega_{2}}+\|\lambda\|_{1 / 2, \Gamma}\right),
$$

where $C$ is a generic constant independent of $h$.

Proof. Let us first define some interpolation operators on $\Omega_{1}$ and $\Omega_{2}$ for the discrete spaces $\mathcal{V}_{1}^{h}$ and $\mathcal{V}_{2}^{h}$. These interpolation operators are straightforwardly adapted from the one defined in [6]. Given a displacement field $u$ satisfying $u_{r}=u-u_{s}$ with $u_{r} \in H^{2}\left(\Omega, \mathbb{R}^{2}\right)$ and denoting

$$
\Omega^{+}=\left\{x \in \Omega,\left(x-x^{*}\right) \cdot n^{0}>0\right\}, \quad \Omega^{-}=\left\{x \in \Omega,\left(x-x^{*}\right) \cdot n^{0}<0\right\},
$$

with the same notations as in (2), we consider the following expressions for $\mathcal{I}_{1}^{h} u$ and $\mathcal{I}_{2}^{h} u$ :

- Let $\tilde{u}^{+}$and $\tilde{u}^{-}$be two extensions of $u^{+}=u_{\left.\right|_{\Omega^{+} \cap \Omega_{1}}}$ and $u^{-}=u_{\left.r\right|_{\Omega^{-} \cap \Omega_{1}}}$ both in $H^{2}\left(\bar{\Omega}_{1} ; \mathbb{R}^{d}\right)$. Then, we consider

$$
\mathcal{I}_{1}^{h} u=\sum_{i \in I\left(\Omega_{1}\right)} a_{i} \varphi_{i}+\sum_{i \in I_{H}\left(\Omega_{1}\right)} b_{i} H \varphi_{i}
$$

where $a_{i}, b_{i}$ are given as follows ( $x_{i}$ denotes the node associated with $\left.\varphi_{i}\right)$ :

$$
\begin{aligned}
& \text { if } i \in\left\{I\left(\Omega_{1}\right) \backslash I_{H}\left(\Omega_{1}\right)\right\} \text { then } a_{i}=u\left(x_{i}\right), \\
& \text { if } i \in I_{H}\left(\Omega_{1}\right) \text { and } x_{i} \in \bar{\Omega}^{\varepsilon} \text { then }\left\{\begin{array}{l}
a_{i}=\frac{1}{2}\left(u^{\varepsilon}\left(x_{i}\right)+\tilde{u}^{-\varepsilon}\left(x_{i}\right)\right), \\
b_{i}=\varepsilon \frac{1}{2}\left(u^{\varepsilon}\left(x_{i}\right)-\tilde{u}^{-\varepsilon}\left(x_{i}\right)\right) .
\end{array}\right.
\end{aligned}
$$

- Let $\tilde{u}_{r}^{+}$and $\tilde{u}_{r}^{-}$be two extensions of $u_{r}^{+}=u_{\left.r\right|_{\Omega+}}$ and $u_{r}^{-}=u_{r \mid \Omega-}$ both in $H^{2}\left(\bar{\Omega} ; \mathbb{R}^{d}\right)$. Then, we consider

$$
\mathcal{I}_{2}^{h} u=\sum_{i \in I\left(\Omega_{2}\right)} a_{i} \varphi_{i}+\sum_{i \in I_{H}\left(\Omega_{2}\right)} b_{i} H \varphi_{i}+u_{s}
$$

where $a_{i}, b_{i}$ are given as follows ( $x_{i}$ denotes the node associated with $\varphi_{i}$ ):

$$
\begin{aligned}
& \text { if } i \in\left\{I\left(\Omega_{2}\right) \backslash I_{H}\left(\Omega_{2}\right)\right\} \text { then } a_{i}=u_{r}\left(x_{i}\right), \\
& \text { if } i \in I_{H}\left(\Omega_{2}\right) \text { and } x_{i} \in \bar{\Omega}^{\varepsilon} \text { then }\left\{\begin{array}{l}
a_{i}=\frac{1}{2}\left(u_{r}^{\varepsilon}\left(x_{i}\right)+\tilde{u}_{r}^{-\varepsilon}\left(x_{i}\right)\right), \\
b_{i}=\varepsilon \frac{1}{2}\left(u_{r}^{\varepsilon}\left(x_{i}\right)-\tilde{u}_{r}^{-\varepsilon}\left(x_{i}\right)\right) .
\end{array}\right.
\end{aligned}
$$

With these definitions we denote $\mathcal{I}^{h} u$ the element of $\mathcal{V}^{h}$ such that:

$$
\mathcal{I}^{h} u=\mathcal{I}_{k}^{h} u_{k} \text { in } \Omega_{k} \text {. }
$$

Using Proposition 6.1, we have

$$
\left\|u-u^{h}\right\|_{\mathcal{V}}^{2}+\left\|\lambda-\lambda^{h}\right\|_{-1 / 2, \Gamma}^{2} \leqslant C\left\{\left\|u-\mathcal{I}^{h} u\right\|_{\mathcal{V}}^{2}+\left\|\lambda-\Pi^{h} \lambda\right\|_{-1 / 2, \Gamma}^{2}\right\},
$$

where $\Pi^{h}$ is the approximation operator defined in Section 7. Then 


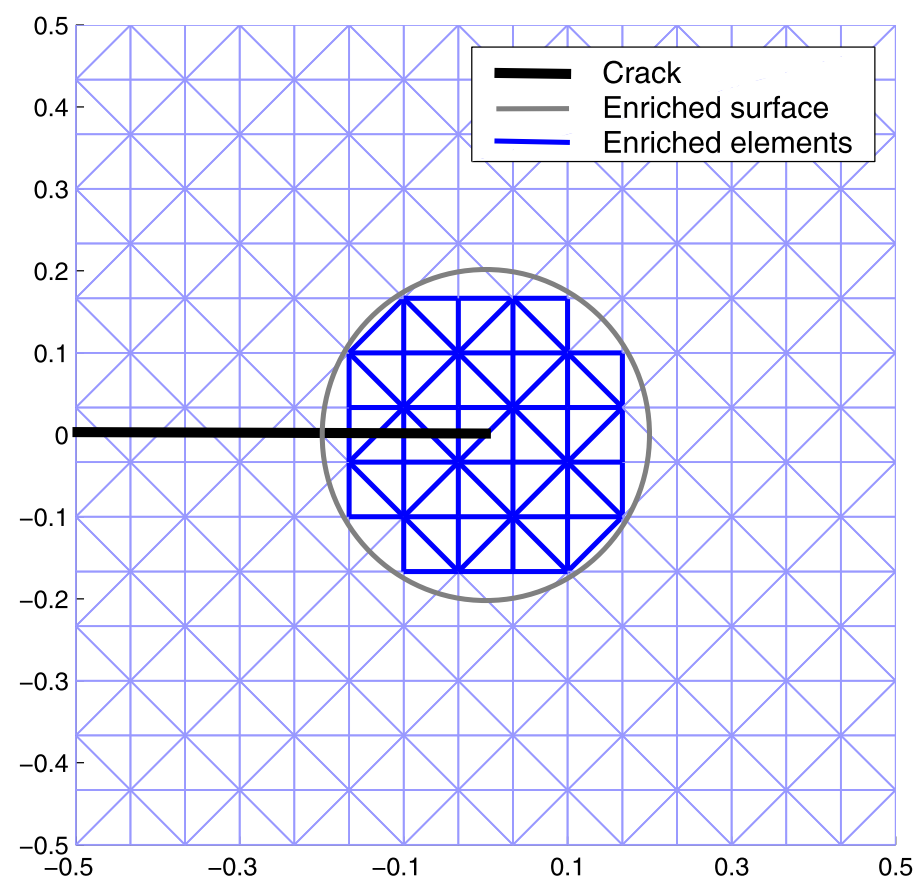

Fig. 5. Regular mesh of a square domain, independent of the crack, and enrichment surface.

$$
\left\|u-u^{h}\right\|_{\mathcal{V}}^{2}+\left\|\lambda-\lambda^{h}\right\|_{-1 / 2, \Gamma}^{2} \leqslant C\left\{\sum_{k \in\{1,2\}}\left\|u-\mathcal{I}_{k}^{h} u_{k}\right\|_{\mathcal{V}_{k}}^{2}+\left\|\lambda-\Pi^{h} \lambda\right\|_{-1 / 2, \Gamma}^{2}\right\} .
$$
that

Now, the optimal results obtained in [22] for Xfem interpolation operators can be applied to $\mathcal{I}_{1}^{h}$ and $\mathcal{I}_{2}^{h}$. We can conclude

$$
\left\|u-\mathcal{I}_{1}^{h} u\right\|_{\mathcal{V}_{1}}^{2} \leqslant C h^{2}\|u\|_{2, \Omega_{1}}^{2}, \quad\left\|u-\mathcal{I}_{2}^{h} u\right\|_{\mathcal{V}_{2}}^{2} \leqslant C h^{2}\left\|u-u_{s}\right\|_{2, \Omega_{2}}^{2}
$$

In addition, the approximation property on multipliers (102) reads

$$
\left\|\lambda-\Pi^{h} \lambda\right\|_{-1 / 2, \Gamma}^{2} \leqslant C h\|\lambda\|_{1 / 2, \Gamma}^{2} .
$$

Consequently, the inequality (114) together with the two latter estimates leads to the result of the theorem.

\section{Numerical experiments}

We consider the cracked domain

$$
\Omega=]-0.5 ; 0.5[\times]-0.5 ; 0.5\left[\backslash \Gamma_{C},\right.
$$

where $\Gamma_{C}$ denotes the crack given by

$$
\Gamma_{C}=[-0.5 ; 0] \times\{0\} .
$$

The Dirichlet condition prescribed on the boundary is the exact opening mode displacement field $u_{I}$ given by (103).

A $P_{1}$ finite element method is considered on a structured mesh of $\bar{\Omega}$ (see Fig. 5). The singular enrichment area is chosen as the set of elements lying in the ball $B\left(x^{*}, 0.2\right)$, where $x^{*}$ denotes the crack tip. An example of such a mesh and a representation of the enrichment surface are shown in Fig. 5. The numerical tests are implemented using Getfem++ [24], the object oriented C++ finite element library developed by our team.

Fig. 6 shows the Von Mises stress on the deformed structure obtained by using Xfem with an integral matching condition on the boundary of the singular enrichment surface. Around the crack tip and on the enriched surface, the level-set curves of the Von Mises are greatly smoother than for the same experiment done with standard Xfem with surface enrichment (and the same enrichment area radius) given in Fig. 7 (see [17]). Xfem with integral matching improves the approximation around the crack tip and at the transition layer between the singular enrichment area and the rest of the domain.

Figs. 8 and 9 show a comparison between the error convergence curves of the classical Xfem with surface enrichment (see [17]), Xfem with a cut-off function (see [6]) and Xfem with integral matching for the $L^{2}$-norm and the $H^{1}$-norm. These errors are computed with respect to the subdivisions number $n s$ in each direction $(n s=1 / h)$. 


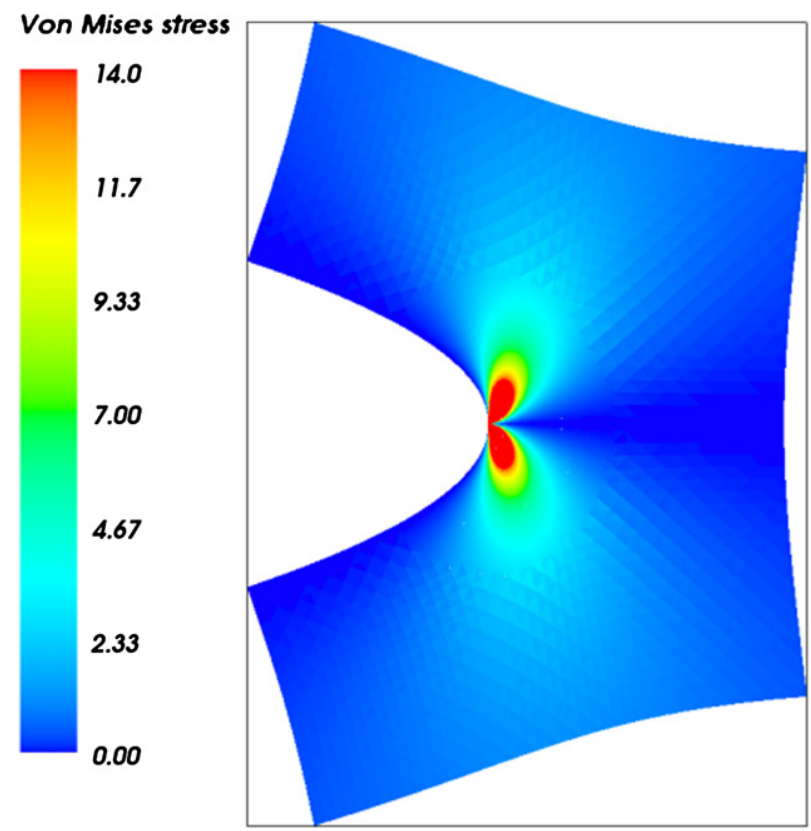

Fig. 6. Von Mises stress for a mode I problem using the integral matching Xfem with $P_{1}$ elements.

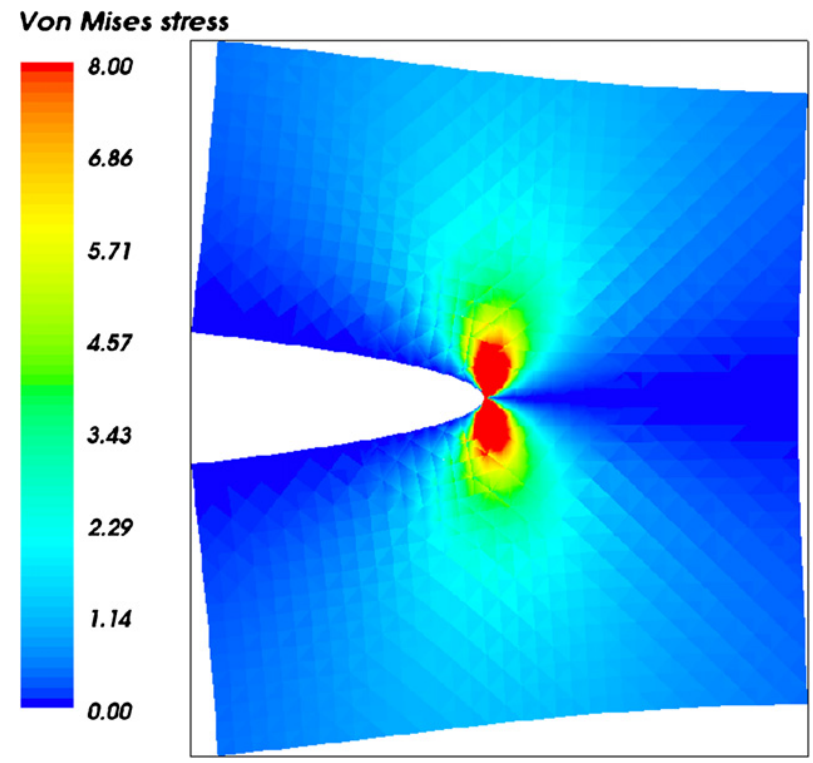

Fig. 7. Von Mises stress for a mode I problem using the surface enrichment Xfem with $P_{1}$ elements.

In Fig. 10, we consider the stress intensity factor $K_{I}$ computed using the $J$-integral and the contour integral for an exact solution given by $P(x)+3 u_{I}+5 u_{I I}$, where $P(x)$ is a given regular solution on the non-cracked domain (see [21] for the computation of the contour integral). The convergence curves for the classical Fem, Xfem with a cut-off function and Xfem with surface enrichment are smooth but overestimate the exact value of $K_{I}$, while Xfem with integral matching is oscillating around this value. Moreover, the Xfem approaches show an optimal convergence rate although the displacement field is singular at the crack tip.

We perform as well mixed mode calculations on the same regular mesh of the cracked square. The considered mixed mode solution is given by $-6 \times P(x)+2 u_{I}-3 u_{I I}$, where $P(x)$ is a given regular solution on the non-cracked domain. Fig. 11 illustrate the corresponding Von Mises stress on the deformed model while Figs. 12 and 13 show a comparison between the error convergence curves of the cutoff Xfem, the integral matching Xfem and the surface enrichment Xfem. 


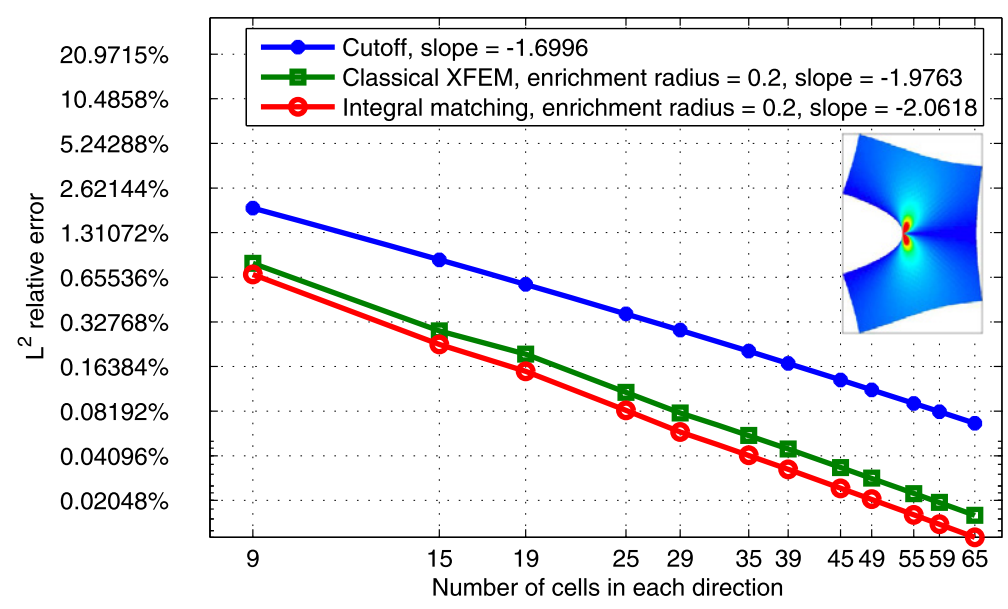

Fig. 8. $L^{2}$-error with respect to the number of cells in each direction for a mode I problem with enriched $P_{1}$ elements (logarithmic scales).

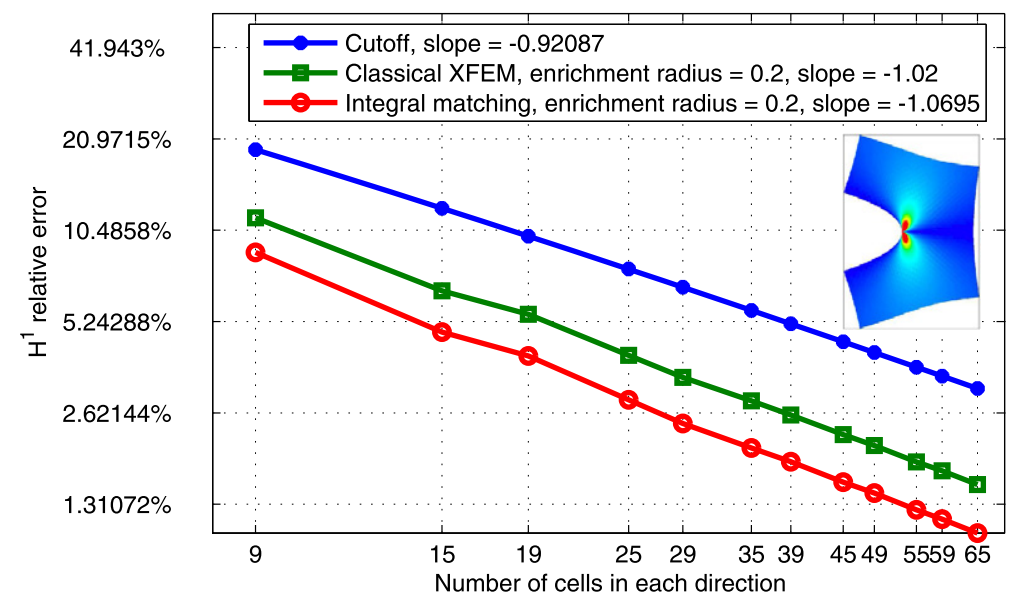

Fig. 9. $H^{1}$-error with respect to the number of cells in each direction for a mode I problem with enriched $P_{1}$ elements (logarithmic scales).

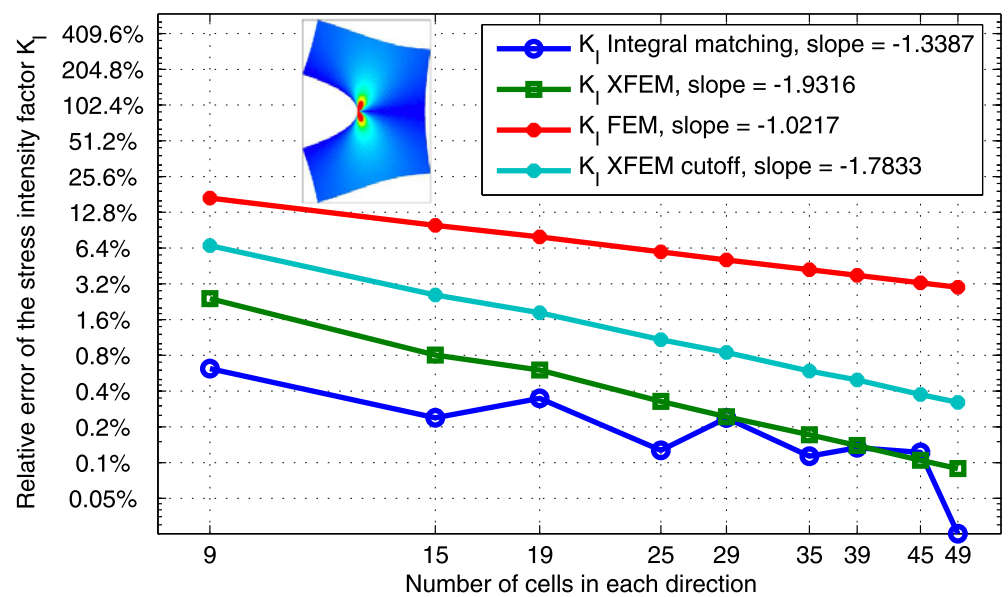

Fig. 10. Computation of the stress intensity factor $K_{I}$ using enriched $P_{1}$ elements: relative error with respect to the number of cells in each direction (logarithmic scales). 


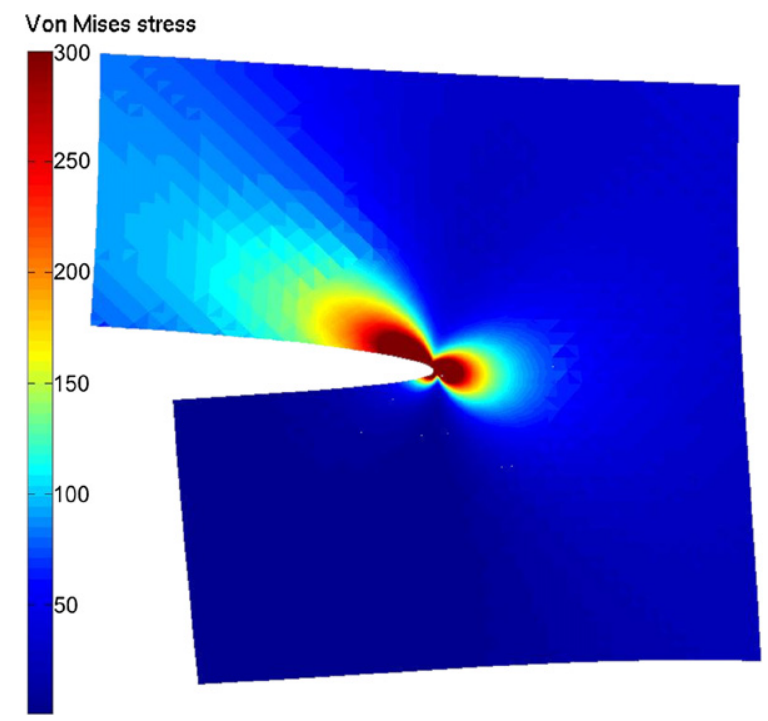

Fig. 11. Von Mises stress for a mode I problem using the integral matching Xfem with $P_{1}$ elements.

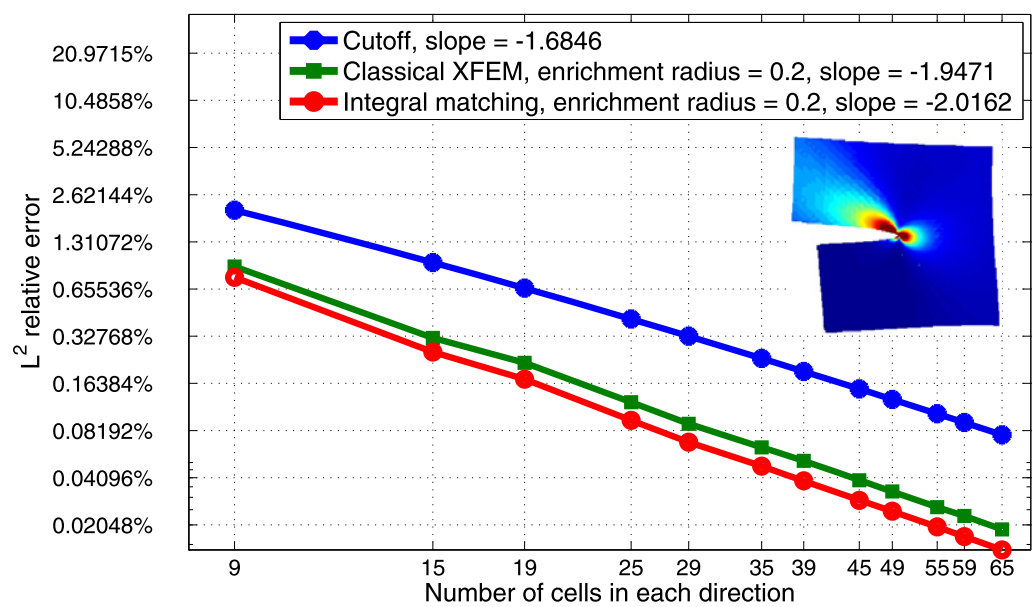

Fig. 12. $L^{2}$-error with respect to the number of cells in each direction for a mixed mode problem on the polygonal domain using enriched $P_{1}$ elements (logarithmic scales).

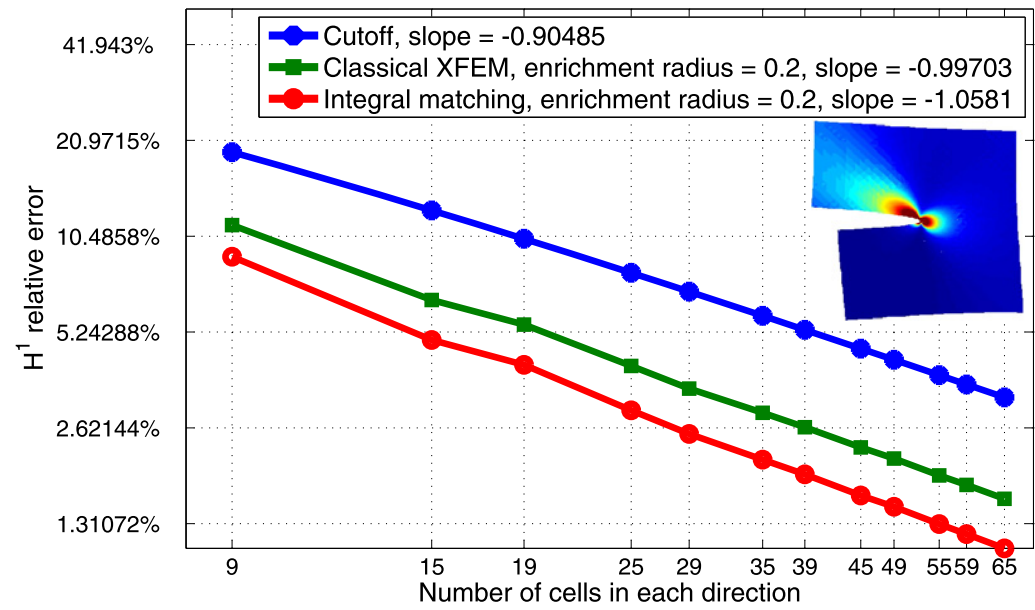

Fig. 13. $H^{1}$-error with respect to the number of cells in each direction for a mixed mode problem on the polygonal domain using enriched $P_{1}$ elements (logarithmic scales). 


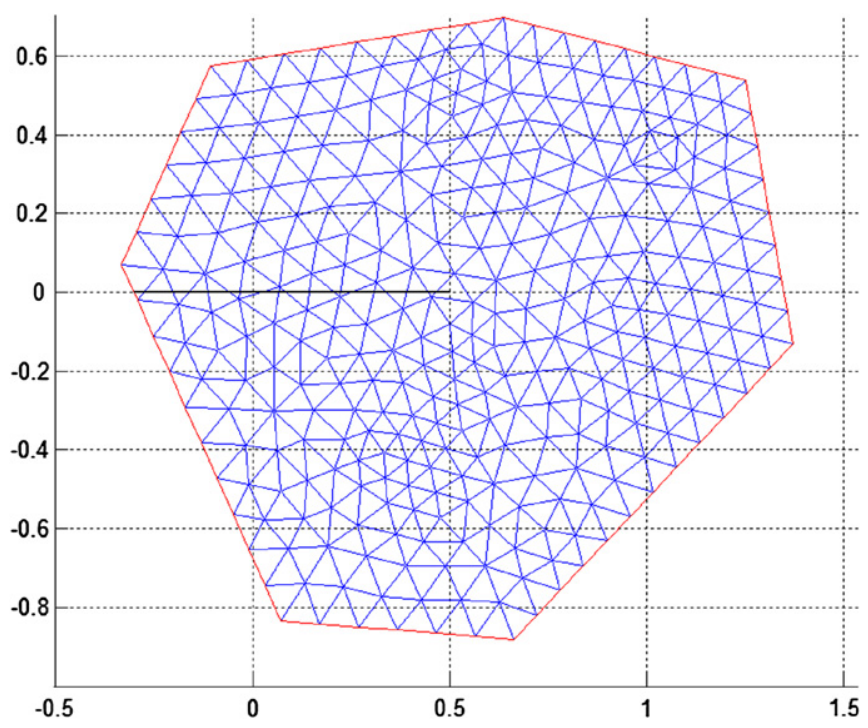

Fig. 14. Non-structured mesh of a polygonal cracked domain.

Table 1

Number of degrees of freedom.

\begin{tabular}{lrrr}
\hline Number of cells in each direction & Fem & Xfem surface enrichment & Xfem cut-off \\
\hline 40 & 3402 & 4962 & 3410 \\
60 & 7508 & 11014 & 7510 \\
80 & 13202 & 19578 & 7656 \\
\hline
\end{tabular}

Now, we consider also a cracked polygonal domain still denoted $\Omega$ (represented in Fig. 14) together with a family of non-structured meshes of $\bar{\Omega}$. The Von Mises stress over the deformed domain is shown in Fig. 15. Figs. 16 and 17 give the $L^{2}$-error and the $H^{1}$-error convergence curves for the classical Xfem with surface enrichment, Xfem with a cut-off function and Xfem with integral matching.

The convergence rate of the three compared strategies is of order $h$ for the energy norm and of order $h^{2}$ for the $L^{2}$ norm, which are the optimal rates (obtained when using a classical finite element method on a regular non-cracked domain). Furthermore, the error values of Xfem with integral matching are lower than those of the two other methods. In fact, the computed error for the cut-off enrichment depends on the norm of the second order derivative of the cut-off function, i.e. depends on the transition layer between the enriched and the non-enriched elements (see [6]). In the classical surface enrichment strategy, there exists also a transition layer defined on the elements partially enriched by the singular functions (see [17]). The integral matching approach removes this layer and replaces it by a non-conformal matching condition on the boundary of the enriched surface. This is pointed out in Fig. 18 that shows the error variation along the vertical line $x=0.1$ for Xfem with a cut-off enrichment, the classical Xfem with surface enrichment and Xfem with integral matching. The latter method offers the lower error around the transition layer (at $y=0.2$ and $y=-0.2$ ). This explains the better accuracy of the approximation noticed in Figs. 8, 9, 16 and 17.

Moreover, Xfem with a cut-off function reduces significantly the computational cost with respect to classical surface enrichment Xfem. Compared with the cut-off method, the integral matching Xfem only adds few degrees of freedom corresponding to the multiplier. Some examples of the evolution of the computational cost for different $P_{1}$ Xfem strategies are shown in Table 1.

On the other hand, since the integral matching is a globalized enrichment strategy, it does not degrade the condition number of the associated linear system (see Fig. 19). Moreover, the sparsity of the stiffness matrix is globally preserved because the singular enrichment is present only in few corresponding lines.

\section{Concluding remarks}

Finally, thanks to the integral bonding condition that removes the transition layer, the quality of the approximation is enhanced with respect to Xfem with surface enrichment and to the cut-off strategy. The mathematical error estimates are optimal and they do not depend neither on a stiff character of the cut-off function nor on a width of the transition layer. This leads to a significant decrease of the error level.

An extension of the proposed method to 3D cracks can be considered but it leads at least to two difficulties: the first one is the determination of the asymptotic displacement near the crack front especially at the boundary of the elastic body (there is only a few situations where the asymptotic displacement is known in 3D). The second one is the definition of a coordinate system along the crack front to introduce a parameterized enrichment. 


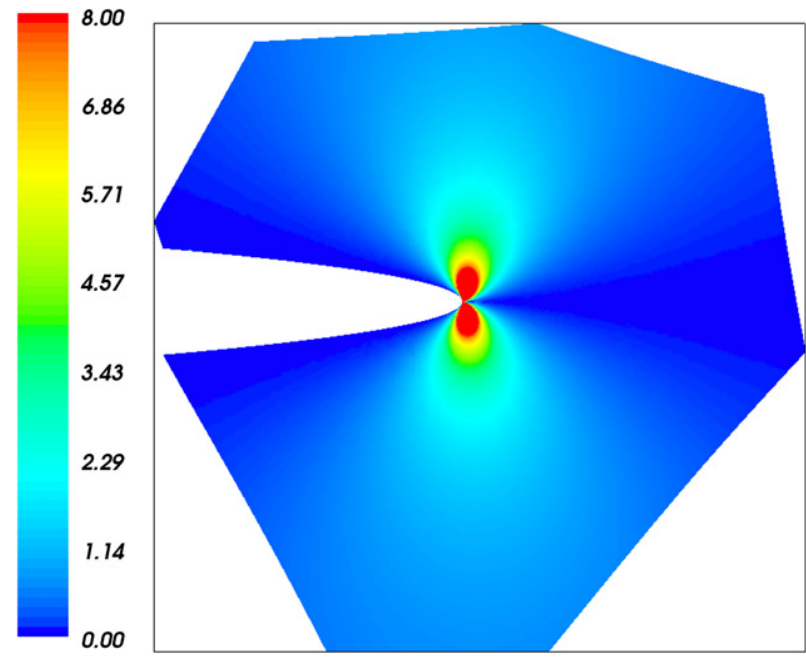

Fig. 15. Von Mises stress for mixed modes using enriched $P_{2}$ elements.

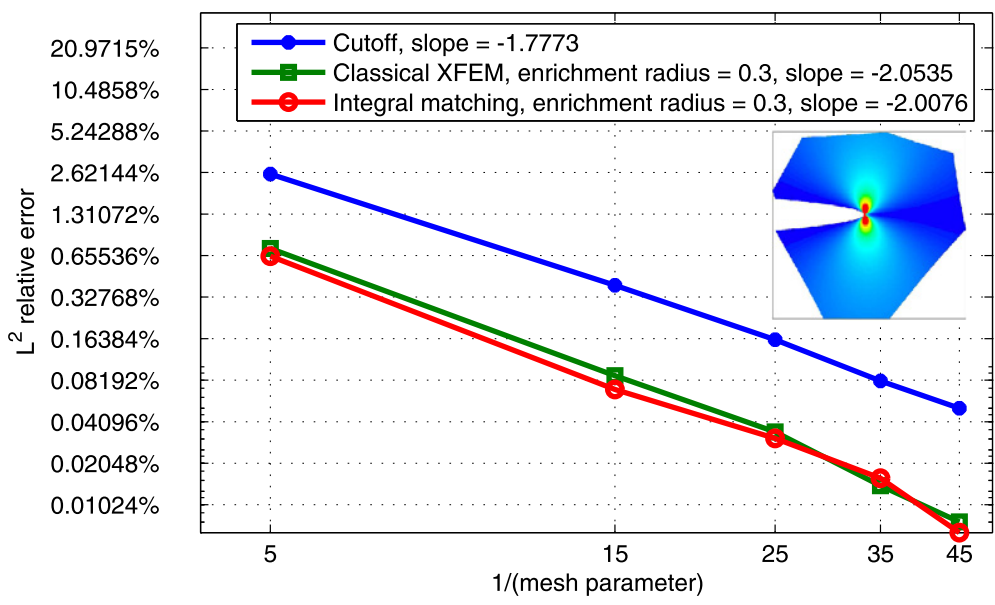

Fig. 16. $L^{2}$-error with respect to the number of cells in each direction for mixed modes problem on the polygonal domain using enriched $P_{1}$ elements (logarithmic scales).

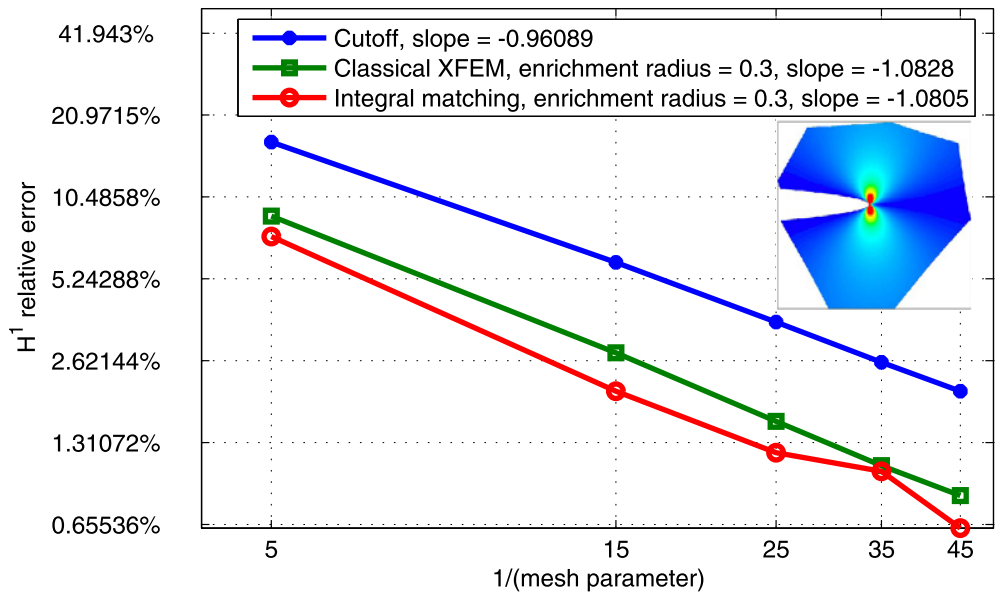

Fig. 17. $H^{1}$-error with respect to the number of cells in each direction for mixed modes problem on the polygonal domain using enriched $P_{1}$ elements (logarithmic scales). 


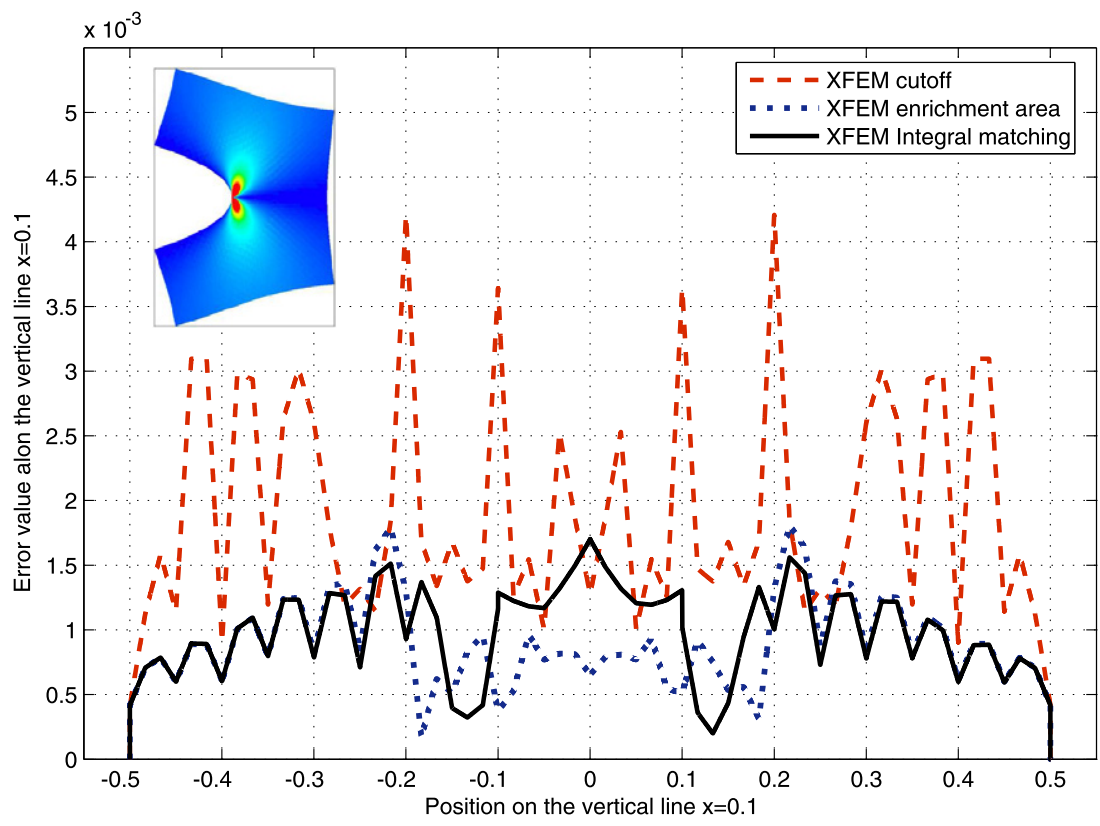

Fig. 18. Error variation along the vertical line $x=0.1$.

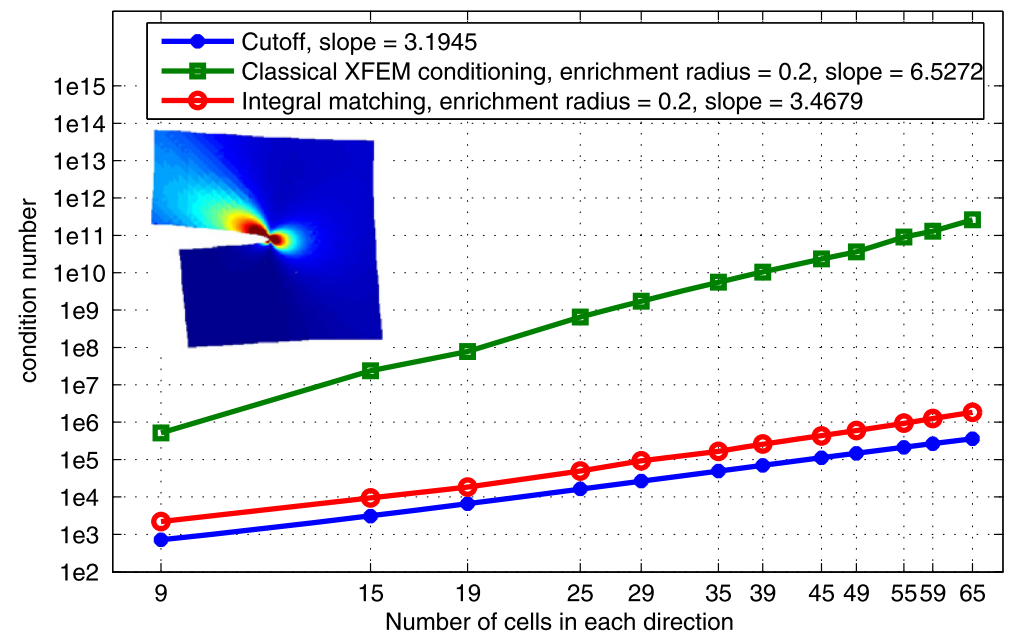

Fig. 19. Condition number of the stiffness matrix with respect to the number of cells in each direction for a mixed mode problem.

\section{Acknowledgements}

The authors would like to thank Julien Pommier for his support to obtain the numerical results and Patrick Hild for some helpfull discussions.

\section{References}

[1] E. Béchet, H. Minnebo, N. Moës, B. Burgardt, Improved implementation and robustness study of the X-fem for stress analysis around cracks, Int. J. Numer. Meth. Engng. 64 (2005) 1033-1056.

[2] F. Ben Belgacem, Y. Renard, Hybrid finite element methods for the Signorini problem, Mathematics of Computations 72 (243) (2003) 1117-1145.

[3] C. Bernardi, Y. Maday, A. Patera, A new nonconforming approach to domain decomposition: the mortar element method, in: Nonlinear Partial Differential Equations and Their Applications, Collège de France Seminar, 1994, pp. 13-51.

[4] F. Brezzi, M. Fortin, Mixed and Hybrid Finite Element Methods, Springer-Verlag, 1991.

[5] E. Chahine, Étude mathématique et numérique de méthodes d'éléments finis étendues pour le calcul en domaines fissurés, PhD thesis, INSA Toulouse, France, 2008.

[6] E. Chahine, P. Laborde, Y. Renard, Crack tip enrichment in the Xfem method using a cut-off function, Int. J. Numer. Meth. Engng. 75 (6) (2008) 629-646.

[7] Z. Chen, R.H. Nochetto, Residual type a posteriori error estimates for elliptic obstacle problems, Numerische Mathematik 84 (2000) 527-548.

[8] J. Chessa, H. Wang, T. Belytschko, On the construction of blending elements for local partition of unity enriched finite elements, Int. J. Numer. Meth. Engng. 57 (2003) 1015-1038. 
[9] P.G. Ciarlet, The Finite Element Method for Elliptic Problems, Studies in Mathematics and Its Applications, vol. 4, North-Holland, 1978.

[10] G. Duvaut, Mécanique des milieux continus, Masson, 1990.

[11] G. Duvaut, J.L. Lions, Les inéquations en mécanique et en physique, Dunod, 1972.

[12] A. Ern, J.-L. Guermond, Éléments finis: théorie, applications, mise en œuvre, Mathématiques et Applications, vol. 36, SMAI, Springer-Verlag, 2002.

[13] T.P. Fries, A corrected XFEM approximation without problems in blending elements, Int. J. Numer. Meth. Engng. 72 (5) (2008) 503-532.

[14] R. Gracie, H. Wang, T. Belytschko, Blending in the extended finite element method by discontinuous Galerkin and assumed strain methods, Int. J. Numer. Meth. Engng. 74 (11) (2008) 1645-1669.

[15] P. Grisvard, Singularities in Boundary Value Problems, Masson, 1992.

[16] P. Hild, Y. Renard, An error estimate for the Signorini problem with Coulomb friction approximated by finite elements, SIAM Journal on Numerical Analysis 45 (5) (2007) 2012-2031.

[17] P. Laborde, Y. Renard, J. Pommier, M. Salaün, High order extended finite element method for cracked domains, Int. J. Numer. Meth. Engng. 64 (2005) 354-381.

[18] A. Legay, H.W. Wang, T. Belytschko, Strong and weak arbitrary discontinuities in spectral finite elements, Int. J. Numer. Meth. Engng. 64 (2005) $991-$ 1008.

[19] J. Lemaitre, J.-L. Chaboche, Mechanics of Solid Materials, Cambridge University Press, 1994.

[20] J.M. Melenk, I. Babuška, The partition of unity finite element method: Basic theory and applications, Comput. Methods Appl. Mech. Engrg. 139 (1996) 289-314.

[21] N. Moës, J. Dolbow, T. Belytschko, A finite element method for crack growth without remeshing, Int. J. Numer. Meth. Engng. 46 (1999) 131-150.

[22] S. Nicaise, Y. Renard, E. Chahine, Optimal convergence analysis for the eXtended Finite Element Method, Int. J. Numer. Meth. Engng., in press.

[23] A. Quarteroni, A. Valli, Domain Decomposition Methods for Partial Differential Equations, Oxford Science Publications, 1999.

[24] Y. Renard, J. Pommier, Getfem++. An open source generic C++ library for finite element methods, http://home.gna.org/getfem.

[25] O. Steinbach, Stability Estimates for Hybrid Coupled Domaine Decomposition Methods, Springer-Verlag, 2003.

[26] J.E. Tarancón, A. Vercher, E. Giner, F.J. Fuenmayor, Enhanced blending elements for XFEM applied to linear elastic fracture mechanics, Int. J. Numer. Meth. Engng. 77 (2009) 126-148.

[27] Q.Z. Xiao, B.L. Karihaloo, Implementation of hybrid crack element on a general finite element mesh and in combination with XFEM, Comput. Methods Appl. Mech. Engrg. 196 (2007) 1864-1873. 